\title{
Perverse consequences of infrequently culling a pest
}

\author{
Alan J. Terry ${ }^{\dagger}$ and Stephen A. Gourley ${ }^{\ddagger}$ \\ ${ }^{\dagger}$ Division of Mathematics, University of Dundee, Dundee, DD1 4HN, UK. \\ ${ }^{\ddagger}$ Department of Mathematics, University of Surrey, Guildford, Surrey, GU2 7XH, \\ UK.
}

All correspondence to Alan J. Terry at above address

E-mail: aterry@maths.dundee.ac.uk

\begin{abstract}
There are potentially many situations in which creatures will be subject to infrequent but regular culling. In terms of controlling crop pests, some farmers may only be able to afford to apply pesticides occasionally. Alternatively, pesticides may be applied only occasionally to limit their unwelcome side effects, which include pesticide resistance, chemical poisoning of agricultural workers, and environmental degradation. In terms of conservation, some species (such as the red deer in the UK) may be culled occasionally to maintain balances within their ecosystem. However, in this paper we discover, as the culmination of an exploration of adult-stage culling of a creature with juvenile and adult life stages, that, in certain circumstances, regular but infrequent culling will, perversely, increase the average population of the creature.
\end{abstract}

Key words: pest control, impulsive adult culling, delay differential equation AMS subject classifications (2000): 34K45, 34K60, 92D25

Short title: Perverse consequences of culling a pest

\section{Introduction}

Creatures can be pests for a variety of reasons. Some insects, such as the colorado potato beetle [1], boll weevil [2], and codling moth [3], feed on crops, whilst certain types of mosquito act as vectors in the spread of human or livestock diseases. Diseases spread by mosquitoes include malaria (which kills over a million people every year), yellow fever, West Nile virus, dengue fever, and various forms of encephalitis $[4,5]$. In the UK, badgers are believed to spread tuberculosis to cattle [6]. Rodents may promote the spread of disease by inhabiting food storage facilities. In terms of conservation, particular kinds of large mammal, including red deer in Scotland [7] and elephants in South Africa [8], can be problematic when they become numerous enough to cause disruption to their ecosystem or habitat.

When creatures act as pests it is common for people to implement control programs. For instance, agricultural insect pests may be culled by the application of pesticides and creatures such as badgers or red deer may be culled by shooting at certain times of the year. Culling is often stage-specific, that is, it is directed against a particular developmental stage in the life cycle of the pest. Adult badgers and red deer are more likely to be targeted than juveniles and insecticides are frequently designed to kill either larvae, in which case they are called larvicides, or adults, in which case they are called adulticides. There are even pesticides which target eggs - these are known as ovicides. Culling need not take place only at discrete times. It can also occur continuously in time but at discrete points in space, as in the trapping of insects by exploiting their attraction to pheromones or other chemicals. 
Such trapping is used to control the Australian blowfly Lucilia cuprina, for example, which is a substantial nuisance to sheep and therefore to sheep farmers [9].

The aim of a pest control program may be localised eradication in the case of a crop pest, or it may simply be to reduce the population of the target creature without wiping it out, as in the culling of red deer with a view to maintaining balances within its ecosystem. Adult deer have no natural predators in the UK or Ireland since wolves were hunted to extinction. It is certainly never the intention of a pest control program to make the pest more abundant. In this paper, however, we will establish that a poorly planned program involving adult impulsive culling may make a pest more successful. (It is of course already known that poorly planned biocontrol programs can have unexpected and even disastrous consequences, such as the introduction of the cane toad in Australia $[10,11]$.)

A number of papers on pest control have appeared in recent years in the literature of mathematical biology. Simons and Gourley [12] found conditions for the eradication of a pest population by adult impulsive culling or by culling adults continuously in time but at discrete points in space. Terry [13] also found conditions for eradication by adult impulsive culling, in particular demonstrating that eradication is possible in only finitely many culls when an Allee effect holds, that is, when there is a minimum viable population.

The adult impulsive culling regimes of Simons and Gourley [12] and of Terry [13] succeed if they are suitably strong. Thus they succeed, for example, if consecutive culls occur sufficiently close together in time. Unfortunately, if the pest is an insect and each cull consists of a dosage of pesticide, then regular frequent culling amounts to a considerable level of pesticide application which may be quite expensive for a farmer and may lead to a number of unwelcome side effects, including pesticide resistance, the loss of the pest's natural predators, secondary pest outbreaks, chemical poisoning of agricultural workers, pesticide residues on food intended for human consumption, and a general depletion of biodiversity [13, 14, 15]. Occasional applications of pesticides might be expected to reduce a pest population whilst limiting expenditure on pesticides as well as potential environmental side effects. Similarly, occasional culls of a creature such as the red deer might be expected to reduce the population without causing it to be eradicated - eradication here would not be the aim of culling. Yet results exist in the literature (in the form of simulations to be described in Section 3) that suggest infrequent but regular culling may, perversely, increase the average population of a pest. To our knowledge, no analytical demonstration of this observation has so far been given. Such a demonstration is given in this paper as the culmination of a wider exploration of the consequences of culling a pest.

The format of this paper is as follows. In Section 2 we describe a general model for a pest with a stage-structured life cycle subject to adult culling. In Section 3 we motivate ourselves by describing simulations in the literature that suggest occasional culling can benefit a pest. In Sections 4 and 5 we derive conditions on a culling regime such that the regime reduces the long time average population of the pest. A determination of the qualitative features of the birth function that potentially permit culling to benefit a pest is carried out in Section 6 . The insight that we thereby gain is used (in Sections 7 and 8) to construct a simple but biologically plausible birth function and a specific culling regime such that an analytical demonstration of the benefit to the pest becomes straightforward. This analytical demonstration is given in Section 9. Simulations in Section 10 corroborate our findings and a discussion ends the paper in Section 11. 


\section{A general model}

We consider a species with a life cycle of two stages, namely juvenile (or immature) and adult. Only the adults can reproduce. (This last assumption is not unnecessary - some types of pest, such as aphids, can be born pregnant! [16].) It is possible to model creatures with more than two life stages by only two if we lump together the pre-adult stages into a single pre-reproductive one.

Suppose that a juvenile becomes an adult upon reaching age $\tau$, assuming it lives that long. Here $\tau$ is a positive constant called the maturation age. Let the juvenile and adult per capita death rates be $\mu_{j}$ and $\mu$ respectively, where $\mu_{j}$ and $\mu$ are positive constants. Also let the adult population at time $t$ be $N(t)$ and suppose that the birth rate at time $t$ is a function of the total number of adults, namely $b(N(t))$. Assume that the juveniles satisfy a standard McKendrick-von Foerster model and that adults are subject to a per capita culling rate $f(t)$. Then, by reasoning as in Section 2 of [12], we find a sensible model for the adult population to be:

$$
\begin{array}{rlrl}
\frac{d N(t)}{d t} & =e^{-\mu_{j} \tau} b(N(t-\tau))-\mu N(t)-f(t) N(t) & & \text { for } t>0 \\
N(t) & =N_{0}(t) \geq 0 \quad \text { for } t \in[-\tau, 0], &
\end{array}
$$

where $N_{0}(t), t \in[-\tau, 0]$ represents prescribed initial data. For a very readable article on the formulation and analysis of this type of delay equation (delay in the coefficients as well as the arguments), see Cooke et al [17]. Here $\mu_{j}$ is assumed to be constant for simplicity, but a similar model can also be derived for the case that $\mu_{j}$ depends on age, resulting in the birth to adult survival probability $e^{-\mu_{j} \tau}$ being replaced by another expression.

The terms in (1) may be interpreted ecologically. The rate at which new adults arise at time $t$ is the rate at which juveniles mature at time $t$. But since the maturation age $\tau$ is constant, the rate at which juveniles mature at time $t$ must be the birth rate at time $t-\tau$ (which is $b(N(t-\tau))$ ), scaled by the proportion of these new borns that survive to maturity (which is $e^{-\mu_{j} \tau}$ ). Also, adults are lost due to natural deaths (at a rate $\mu$ ) and due to culling (at a rate $f(t)$ ).

Henceforth we shall frequently refer to $N(t)$ as the "population", even though, technically, it is only the adult population of the creature being studied. A commonly used form for a birth function is $b(N)=\lambda_{1} N e^{-\lambda_{2} N}$ for positive constants $\lambda_{1}$, $\lambda_{2}$. Certain blowfly population experiments by Nicholson $[18,19]$ inspired Gurney et al [20] to consider a birth function of this form, which motivated Terry [13] to label such a function as being of Nicholson-type. However, notice that a function of the form $N e^{r-k N}$, for positive constants $r, k$, is sometimes referred to as a Ricker functional form, descending from fisheries research by Ricker [21, 22].

An example of a Nicholson-type birth function is plotted in figure 2 (right). A Nicholson birth function increases linearly for small $N$ but, as $N$ increases, it reaches a maximum and then decreases to zero. These facts capture the idea that a small population can breed quickly until it grows enough that factors such as overcrowding or competition hinder mating.

Simons and Gourley [12] considered a model with the term

$$
\sum_{i=1}^{\infty} b_{i} N\left(t_{i}^{-}\right) \delta\left(t-t_{i}\right)
$$

in place of $f(t) N(t)$ in (1). Their aim was to consider the case in which culling is an impulsive phenomenon, occurring only at certain particular times $t_{i}$ with $0<t_{1}<t_{2}<\cdots$ and $t_{i} \rightarrow \infty$ as $i \rightarrow \infty$. At the cull which occurs at time $t_{i}$ a proportion $b_{i} \in[0,1]$ of the adult population is culled, causing a sharp decrease 
in the population and consequently a discontinuity in the evolution at time $t_{i}$. Expression (3) is a particular case of $f(t) N(t)$, arising when

$$
f(t)=\sum_{i=1}^{\infty} b_{i} \delta\left(t-t_{i}\right) .
$$

Note in particular that (4) is periodic if the times $t_{i}$ are equally spaced and the $b_{i}$ are all equal. Terry [13] has also recently considered impulsive culling in (1). For an impulsive culling regime, we could intuitively think of the culls as being "infrequent" if each cull were to occur more than $\tau$ time units after the previous one. This would allow an entire generation to grow up and start reproducing between any two consecutive culls.

Our aim in this paper is primarily to study the effect of periodic culling on the long time mean of the population $N(t)$ and in particular to show that in certain biologically plausible circumstances it may actually increase the mean. With this in mind, we shall often make the following set of assumptions on the function $b(\cdot)$, although they are not needed for all of the results:

$$
\begin{aligned}
& b(0)=0, b(N)>0 \text { when } N>0 \text {, there exists } N_{m}>0 \text { such that } \\
& b(N) \text { is increasing for } N \in\left(0, N_{m}\right) \text { and } b(N) \text { is decreasing for } \\
& N>N_{m} \text {; moreover there exists } N^{*}>0 \text { such that } e^{-\mu_{j} \tau} b(N)>\mu N \\
& \text { for } N \in\left(0, N^{*}\right) \text { and } e^{-\mu_{j} \tau} b(N)<\mu N \text { for } N>N^{*} .
\end{aligned}
$$

Note that $N^{*}$ is an equilibrium of (1) in the case when $f(t)=0$. It satisfies $e^{-\mu_{j} \tau} b\left(N^{*}\right)=\mu N^{*}$. Note also that $N^{*}$ could be less than $N_{m}$ or greater than $N_{m}$. The distinction between the two possibilities is important. Also $N^{*}$ depends on the delay $\tau$ while $N_{m}$ does not.

It is easy to see that the assumptions in (5) are biologically sensible. As a small population increases, the birth rate may be expected to increase because the number of breeding individuals increases. However, when a population has grown sufficiently, there will be competition for food and to find a mate, which will hinder reproduction and cause the birth rate to fall. A Nicholson-type birth function (defined above) satisfies (5) for suitable values of its parameters.

Proposition 1. In model (1) suppose that $b(0)=0$ and $b(N)>0$ when $N>0$. Then $N(t) \geq 0$ for all $t>0$. If $N_{0}(s)$ is continuous and $N_{0}(s) \not \equiv 0$ on $[-\tau, 0]$ then there exists $t_{0} \in[0, \tau]$ such that $N(t)>0$ for all $t \geq t_{0}$.

Proof. Theorem 5.2.1 on page 81 of Smith [23] immediately yields that $N(t) \geq 0$ for all $t>0$. For the existence of $t_{0}$, assume for contradiction that $N(t) \equiv 0$ on $[0, \tau]$. Then it follows from (1) that $b(N(t-\tau)) \equiv 0$ on $[0, \tau]$ so that $N(t-\tau) \equiv 0$ on $[0, \tau]$ and therefore $N_{0}(s) \equiv 0$ which is a contradiction. So there exists $t_{0} \in[0, \tau]$ such that $N\left(t_{0}\right)>0$. To prove that $N(t)>0$ for all $t>t_{0}$ note that $N^{\prime}(t) \geq$ $-\mu N(t)-f(t) N(t)$ for all $t>0$, so by a standard comparison argument, for any $t>t_{0}, N(t) \geq \tilde{N}(t)$ where $\tilde{N}^{\prime}(t)=-\mu \tilde{N}(t)-f(t) \tilde{N}(t)$ and $\tilde{N}\left(t_{0}\right)=N\left(t_{0}\right)>0$. Clearly $\tilde{N}(t)>0$ for all $t>t_{0}$, and therefore also $N(t)>0$ for all $t>t_{0}$.

Throughout this paper we shall frequently refer to the long time average or long time mean $\bar{N}$ of a function $N$, which is defined by

$$
\bar{N}=\lim _{t \rightarrow \infty} \frac{1}{t} \int_{0}^{t} N(s) d s .
$$

For brevity we will sometimes refer to the long time average simply as the "mean". 


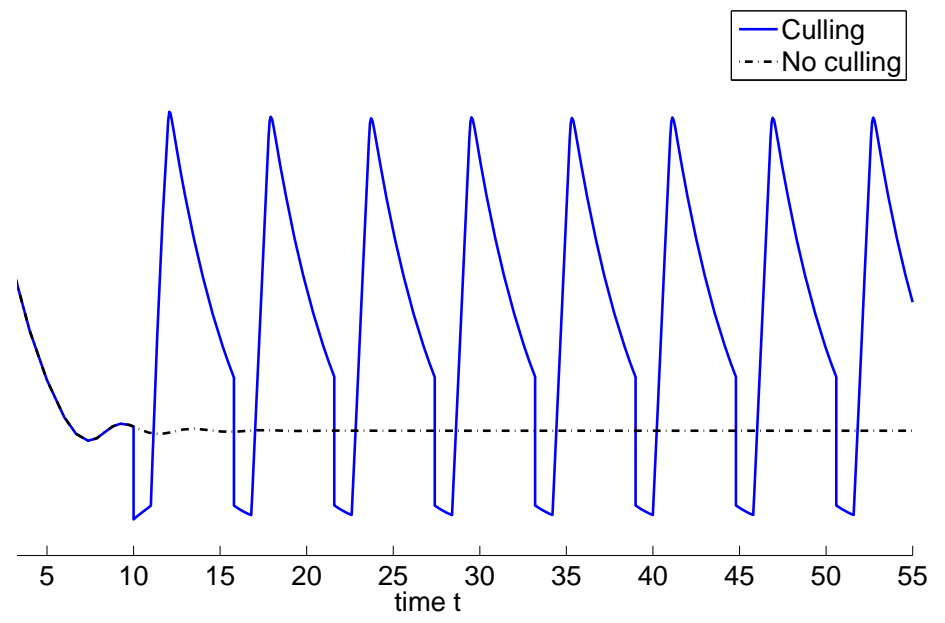

Figure 1: Infrequent culling simulation. Here we simulate $N^{\prime}(t)=e^{-\mu_{j} \tau} b(N(t-$ $\tau))-\mu N(t)-f(t) N(t)$ where $f(t)$ satisfies (4). The birth function is $b(N)=$ $\lambda_{1} N e^{-\lambda_{2} N}$ with $\lambda_{1}$ chosen such that $e^{-\mu_{j} \tau} \lambda_{1}=75$, and $\lambda_{2}=0.4$. The initial data is $N(t)=1$ for $t \in[-\tau, 0]$ where $\tau=1$. The adult death rate is $\mu=0.25$. Culls begin at time $t_{1}=10$ and occur thereafter every $T=5.8$ time units. The cull strength is constant: at the $i$-th cull $(i \geq 1)$ a proportion $b_{i}=0.72$ of the adult population is culled.

\section{Motivation}

A model for the spread of a vector-born infectious disease, with West Nile virus as an application, has been analysed by Gourley et al [24]. The vector in their model has a stage-structured life cycle very similar to the creature described in the last section. A simulation in their paper (see figure 4 in [24]) shows that regular impulsive culling of the adult stage can significantly increase the average vector population if culls occur much less often than every $\tau$ time units (where $\tau$ is the maturation age). Gourley et al [24] intimate that this behaviour can be explained in terms of the shape of the birth function.

Now consider the model (1) where culling is carried out impulsively, so that $f(t)$ satisfies (4), and where the birth function is of Nicholson-type. We include a simulation (figure 1) showing that impulsive culling can increase the average of the population when, again, culls occur much less often than every $\tau$ time units. This behaviour shall be explained in terms of the shape of the birth function in section 6 , which will then allow us to find a simple model for which we may prove that infrequent culling can increase the average population. Before that, we shall establish conditions such that culling will reduce the mean.

\section{Mean reduced in absence of delay}

In this section we consider (1) in the case when $\tau=0$, that is, we consider

$$
N^{\prime}(t)=b(N(t))-\mu N(t)-f(t) N(t), \quad N(0)=N_{0} \geq 0 .
$$

By setting $\tau=0$ we lose the stage-structure of our population and thus we restrict to situations in which the pre-adult stages can be neglected, which is perhaps justifiable if they are of very short duration. 
For the model in (7), we shall prove a theorem that provides two alternative conditions for the solution to approach zero, then prove a theorem on the existence of a periodic solution for the case when $f(t) \geq 0$ is periodic, and then show that periodic culling always lowers the long time mean when delay is absent.

Theorem 1. In model (7) suppose that $f(t) \geq 0$. Then either of the following conditions is sufficient to ensure that $N(t) \rightarrow 0$ as $t \rightarrow \infty$ :

(i) $b(0)=0$ and $b(N)<\left(\mu+f_{\text {inf }}\right) N$ for all $N>0$, where $f_{\inf }=\inf _{t \in[0, \infty)} f(t)$;

(ii) $f(t)$ is periodic of period $T, b(0)=0, b(N)$ is differentiable with $b^{\prime}(N) \leq b^{\prime}(0)$ for all $N>0$, and

$$
b^{\prime}(0)<\mu+\frac{1}{T} \int_{0}^{T} f(t) d t .
$$

Proof. For case (i) note, from (7), that

$$
N^{\prime}(t) \leq b(N(t))-\mu N(t)-f_{\text {inf }} N(t) .
$$

Therefore $N(t)$ is bounded above by the solution satisfying the initial condition for $N$ of the corresponding differential equation obtained by replacing $\leq$ by $=$ in the above. Under the stated conditions this solution tends to zero as $t \rightarrow \infty$, and therefore so does $N(t)$.

To prove (ii) introduce the new variable $x(t)$ defined by $N(t)=e^{x(t)}$. We need to prove that $x(t) \rightarrow-\infty$. Also, write the periodic function $f(t)$ as $f(t)=\bar{f}+f_{0}(t)$ where $f_{0}(t)$ has mean zero and $\bar{f}$ is the mean of $f(t)$, given by $\bar{f}=(1 / T) \int_{0}^{T} f(t) d t$. Then $x(t)$ satisfies

$$
\begin{aligned}
x^{\prime}(t) & =\frac{b\left(e^{x(t)}\right)}{e^{x(t)}}-\mu-\bar{f}-f_{0}(t) \\
& =b^{\prime}(\theta(t))-\mu-\bar{f}-f_{0}(t)
\end{aligned}
$$

for some $\theta(t) \in\left(0, e^{x(t)}\right)$. Integrating from 0 to $n T$ where $n \in \mathbb{N}$, and using that $f_{0}(t)$ has mean zero,

$$
\begin{aligned}
x(n T)-x(0) & =\int_{0}^{n T} b^{\prime}(\theta(s)) d s-\mu n T-\bar{f} n T \\
& \leq \underbrace{\left(b^{\prime}(0)-\mu-\bar{f}\right)}_{<0} n T
\end{aligned}
$$

so that $x(n T) \rightarrow-\infty$ as $n \rightarrow \infty$. For $t>0$ let $n_{t}$ be the integer such that $n_{t} T \leq t<\left(n_{t}+1\right) T$. Then

$$
\begin{aligned}
x(t) & =x\left(n_{t} T\right)+\int_{n_{t} T}^{t} b^{\prime}(\theta(s)) d s-(\mu+\bar{f})\left(t-n_{t} T\right)-\int_{n_{t} T}^{t} f_{0}(s) d s \\
& \leq x\left(n_{t} T\right)+\underbrace{\left(b^{\prime}(0)-\mu-\bar{f}\right)}_{<0}\left(t-n_{t} T\right)+\int_{0}^{T}\left|f_{0}(s)\right| d s .
\end{aligned}
$$

Since $\lim _{t \rightarrow \infty} x\left(n_{t} T\right)=-\infty$, it follows that $\lim _{t \rightarrow \infty} x(t)=-\infty$.

Theorem 2. In model (7) suppose that $f(t) \geq 0$ is non-constant and periodic of period $T$, and that (5) holds with $\tau=0$. Suppose also that $b(\cdot)$ is differentiable and that

$$
b^{\prime}(0)>\mu+f_{\max }
$$

where $f_{\max }=\max _{t \in[0, T]} f(t)$. Then (7) has a non-constant periodic solution of period $T$. 
Proof. We will first prove that if $N(0)>0$ then $N(t)$ remains bounded away from zero for all times. There exists $\delta>0$ independent of $t$ such that, when $N \in(0, \delta]$,

$$
b(N)-\mu N-f(t) N>0
$$

for any $t \geq 0$. This is because $b(N)-\mu N-f(t) N \geq\left(b^{\prime}(0)-\mu-f_{\max }\right) N+O\left(N^{2}\right)$. We claim that $N(t) \geq \delta$ for all $t$ sufficiently large. Strict positivity of $N(t)$ throughout any initial transient is assured by Proposition 1 . There are two cases to consider: $N(0) \geq \delta$ and $N(0)<\delta$. If $N(0) \geq \delta$ then we claim that $N(t) \geq \delta$ for all $t>0$. Suppose that $N(t)$ gets below $\delta$ and let $t^{*}=\inf \{t: N(t)<\delta\}$. Then $N\left(t^{*}\right)=\delta$ and $N^{\prime}\left(t^{*}\right) \leq 0$. However,

$$
N^{\prime}\left(t^{*}\right)=b\left(N\left(t^{*}\right)\right)-\mu N\left(t^{*}\right)-f\left(t^{*}\right) N\left(t^{*}\right)>0
$$

by (9). This is a contradiction. If $N(0)<\delta$ then we claim that there exists $\hat{t}>0$ such that $N(\hat{t}) \geq \delta$. If this is false then $N(t)<\delta$ for all $t \geq 0$, but then

$$
N^{\prime}(t)=b(N(t))-\mu N(t)-f(t) N(t)>0
$$

for all $t \geq 0$, by (9). Therefore $N(t)$ is increasing and bounded above (by $\delta$ ), and therefore tends to some limit $N^{\infty} \in(0, \delta]$. We now claim that $N^{\prime}(t) \rightarrow 0$ as $t \rightarrow \infty$. Since $N(t)$ converges, a necessary and sufficient condition to have $N^{\prime}(t) \rightarrow 0$ is that $N^{\prime}(t+h)-N^{\prime}(t) \rightarrow 0$ as $t \rightarrow \infty, h \rightarrow 0$ (see Corollary A.17 of Thieme [25]). This condition does indeed hold since, from (7),

$$
\begin{aligned}
N^{\prime}(t+h)-N^{\prime}(t)= & b(N(t+h))-b(N(t))-\mu(N(t+h)-N(t)) \\
& -f(t+h)(N(t+h)-N(t))+(f(t)-f(t+h)) N(t) .
\end{aligned}
$$

As $t \rightarrow \infty$ and $h \rightarrow 0$ we have $N(t) \rightarrow N^{\infty}$ and the above expression goes to zero, noting that the last term is bounded in absolute value by $h \max _{t \in[0, T]}\left|f^{\prime}(t)\right| N(t)$. So $N^{\prime}(t) \rightarrow 0$ as $t \rightarrow \infty$. But then (7) implies that $f(t)$ approaches a constant as $t \rightarrow \infty$, which is impossible.

Having established the existence of a $\hat{t}>0$ such that $N(\hat{t}) \geq \delta$ it is clear that $N(t) \geq \delta$ for all $t>\hat{t}$, because the problem is without delay and so the previous case now applies, with $\hat{t}$ playing the role of 0 .

We have shown that the solution is bounded away from zero, if $N(0)>0$. It is also bounded above, since positivity of $f(t)$ and $N(t)$ imply that

$$
N^{\prime}(t) \leq b(N(t))-\mu N(t)
$$

so that $\lim \sup _{t \rightarrow \infty} N(t) \leq N^{*}$. Moreover if $N(0) \leq N^{*}$ then $N(t) \leq N^{*}$ for all $t>0$. In summary, we have shown that the interval $\left[\delta, N^{*}\right]$ is invariant for solutions of (7).

Now consider the family of solutions of (7) parametrised by $\lambda$ :

$$
\mathcal{F}=\left\{N_{\lambda} \text { satisfying }(7) \text { subject to } N_{\lambda}(0)=(1-\lambda) \delta+\lambda N^{*}: 0 \leq \lambda \leq 1\right\},
$$

that is, solutions with initial values in $\left[\delta, N^{*}\right]$. We will show that within this family there is a periodic solution. To see this consider the function $\Phi:[0,1] \rightarrow \mathbb{R}$ defined by

$$
\Phi(\lambda)=N_{\lambda}(T)-N_{\lambda}(0) .
$$

Since $\left[\delta, N^{*}\right]$ is invariant, $N_{\lambda}(T) \in\left[\delta, N^{*}\right]$ for all $\lambda \in[0,1]$. Moreover

$$
\begin{aligned}
& \Phi(1)=N_{1}(T)-N_{1}(0)=N_{1}(T)-N^{*} \leq 0, \\
& \Phi(0)=N_{0}(T)-N_{0}(0)=N_{0}(T)-\delta \geq 0,
\end{aligned}
$$


so that there exists a $\lambda \in[0,1]$ with $\Phi(\lambda)=0$, so that $N_{\lambda}(T)=N_{\lambda}(0)$. Since the differential equation is first order and we are considering the non-delay case with $\tau=0$, this is sufficient to ensure that the solution with initial value $(1-\lambda) \delta+\lambda N^{*}$, and this particular $\lambda$, is periodic. The periodic solution is non-constant because, if it were constant then the differential equation shows that $f(t)$ would also have to be constant.

The above proof (for Theorem 2) establishes the existence of a periodic solution with values in $\left[\delta, N^{*}\right]$. Since $N^{*}$ is the long time mean of the solution in the absence of culling, periodic culling reduces the mean. But in fact any solution in the absence of delay satisfies limsup $\sup _{t \rightarrow \infty} N(t) \leq N^{*}$ so that culling with any non-negative $f(t)$ reduces the mean. In particular this will be the case for impulsive culling, periodic or otherwise, that is described by (4). The analysis of this section shows that culling can only possibly increase the mean if delay is present. It turns out that, even with delay present, there are other conditions that must be met including the requirement that $N^{*}$ be in the interval of values of $N$ for which $b(N)$ is decreasing.

\section{$5 \quad$ Mean reduced in presence of delay}

We now return to (1). Our first result for (1) provides conditions sufficient for the existence of periodic solutions. We first need to introduce some notation. Suppose that $X$ is a real Banach space and that $C \subset X$ is a cone, that is, a nonempty closed subset of $X$ with the properties (i) $\lambda C \subset C$ for any non-negative $\lambda$, (ii) $C+C \subset C$, and (iii) $C \cap(-C)=\{0\}$. Note that such a set is necessarily convex. A total cone is one with the additional property that $\overline{C-C}=X$, where $C-C=\{u-v: u, v \in C\}$.

Recall that a cone induces a partial ordering on a Banach space. We say that $x \leq y$ if and only if $y-x \in C$. We define $x<y$ to mean that $x \leq y$ and $x \neq y$, and we say that $x \ll y$ if and only if $y-x \in \operatorname{int} C$, the interior of $C$.

An operator $T: X \rightarrow X$ is said to be positive if $T(C) \subset C$ (i.e. $T u \geq 0$ when $u \geq 0$ ), and strongly positive if $T(C \backslash\{0\}) \subset \operatorname{int} C$ (i.e. $T u \gg 0$ when $u>0$ ).

We will need the following version of the Krein-Rutman theorem, which combines the relevant parts of the two versions to be found on pages 226 and 228 of Deimling [26]:

Theorem 3 (Krein-Rutman theorem). Let $X$ be a Banach space and let $C \subset X$ be a total cone, and $T: X \rightarrow X$ a compact linear operator that is positive. Suppose that the spectral radius $\rho(T)$ of $T$ satisfies $\rho(T)>0$. Then $\rho(T)$ is an eigenvalue of $T$ with an eigenvector $u \in C \backslash\{0\}$ (i.e. $u>0$ ).

Moreover if $T$ is strongly positive then $\rho(T)$ is a simple eigenvalue, $u \in \operatorname{int} C$ (i.e. $u \gg 0$ ) and there is no other eigenvalue with a positive eigenvector.

In our application $X$ will be taken to be a space of continuous functions, and $C$ the cone of non-negative continuous functions. In this way we define a partial ordering in a function space. We will prove the following theorem which admits only monotone increasing concave $b(\cdot)$.

Theorem 4. In equation (1) let $f(t)$ be periodic of period $T$. Suppose that $b(0)=0$, $b^{\prime}(N)>0$ for all $N>0, \lambda b(N)<b(\lambda N)$ when $\lambda \in(0,1)$ and $N>0$, and $b(\cdot)$ is bounded. Then either every solution of (1) tends to zero as $t \rightarrow \infty$, or (1) has a $T$-periodic solution which is strictly positive at all times, and this solution attracts all solutions with initial data such that $N_{0}(s) \not \equiv 0$ on $[-\tau, 0]$. The latter alternative occurs when the spectral radius of $D F(0)$ strictly exceeds 1 , where $F$ is the operator which maps $N([-\tau, 0])$ to $N([T-\tau, T])$. 
Proof. The proof will be via the use of Theorem 2.3.4 on page 48 of Zhao [27]. Let $X=C[-\tau, 0]$, the Banach space of continuous real valued functions on $[-\tau, 0]$ with the supremum norm

$$
\|N\|=\sup _{s \in[-\tau, 0]}|N(s)|
$$

and let $C$ be the cone of non-negative functions in $X$. If $N(\cdot)$ is a given function defined on $[-\tau, \infty)$ let $N_{t}:[-\tau, 0] \rightarrow \mathbb{R}$ be the function defined by $N_{t}(\theta)=N(t+\theta)$, $\theta \in[-\tau, 0]$. Note in particular that the function $N_{T}$ is defined in terms of the values of $N(\cdot)$ on the interval $[T-\tau, T]$. Now define the operator $F$ to be the Poincaré map of equation (1), so that

$$
F\left(N_{0}\right)=N_{T}
$$

where $N_{T}(\theta)=N(T+\theta), \theta \in[-\tau, 0]$, and $N(t)$ is the solution of (1) corresponding to the initial datum $N_{0}(\theta)=N(\theta), \theta \in[-\tau, 0]$. Thus, with a translation in time, $F\left(N_{0}\right)$ is basically the solution of (1) on the interval $t \in[T-\tau, T]$ that arises from the use of $N_{0}$ as initial datum. A fixed point of $F$ corresponds to a periodic solution of (1) of period $T$.

Clearly $F: C \rightarrow C$; this follows from non-negativity of solutions (Proposition 1). Note also that $F(0)=0$ because the solution of (1) corresponding to zero initial data is the zero solution. Next we shall show that $F$ is strictly subhomogeneous in the sense of Zhao [27], i.e. that $F(\lambda N)>\lambda F(N)$ for any $N \in C$ with $N \gg 0$ and $\lambda \in(0,1)$. Now, $N \gg 0$ means that $N \in \operatorname{int} C$ and therefore that $\inf _{s \in[-\tau, 0]} N(s)>$ 0 . What we need to check is that under these circumstances the solution $N(t ; \lambda)$ of

$$
\begin{aligned}
& N^{\prime}(t ; \lambda)=e^{-\mu_{j} \tau} b(N(t-\tau ; \lambda))-\mu N(t ; \lambda)-f(t) N(t ; \lambda), \\
& N(s ; \lambda)=\lambda N_{0}(s), \quad s \in[-\tau, 0]
\end{aligned}
$$

satisfies

$$
N(t ; \lambda) \geq \lambda N(t) \quad \text { and } \quad N(t ; \lambda) \not \equiv \lambda N(t) \quad \text { on }[T-\tau, T]
$$

where $N(t)$ satisfies

$$
N^{\prime}(t)=e^{-\mu_{j} \tau} b(N(t-\tau))-\mu N(t)-f(t) N(t), \quad N(s)=N_{0}(s), s \in[-\tau, 0] .
$$

Multiplying (13) by $\lambda$ and subtracting the result from (11), and introducing $w(t)=$ $N(t ; \lambda)-\lambda N(t)$, gives $w(s) \equiv 0$ for $s \in[-\tau, 0]$ and, for $t>0$,

$$
\begin{aligned}
w^{\prime}(t) & =e^{-\mu_{j} \tau}(b(N(t-\tau ; \lambda))-\lambda b(N(t-\tau)))-\mu w(t)-f(t) w(t) \\
& \geq e^{-\mu_{j} \tau}(b(N(t-\tau ; \lambda))-b(\lambda N(t-\tau)))-\mu w(t)-f(t) w(t) \\
& =w(t-\tau) e^{-\mu_{j} \tau} b^{\prime}(\theta(t))-\mu w(t)-f(t) w(t)
\end{aligned}
$$

where we have used $\lambda b(N) \leq b(\lambda N)$, and where $\theta(t)$ is a function which arises through an application of the mean value theorem. Since $b^{\prime}(\cdot)>0$ a comparison theorem is applicable and it then follows from Theorem 5.2.1 on page 81 of Smith [23] that $w(t) \geq 0$ for $t \geq 0$ and therefore in particular that $N(t ; \lambda) \geq \lambda N(t)$ on $[T-\tau, T]$. If it were true that $N(t ; \lambda) \equiv \lambda N(t)$ on $[T-\tau, T]$, then it would follow that $w(t) \equiv 0$ on $[T-\tau, T]$ and that

$$
b(N(t-\tau ; \lambda)) \equiv \lambda b(N(t-\tau))
$$

for all $t \in[T-\tau, T]$. But then $b(N(t-\tau ; \lambda)) \leq b(\lambda N(t-\tau))$ so that, by monotonicity of $b(\cdot), N(t-\tau ; \lambda) \leq \lambda N(t-\tau)$ and so $w(t-\tau) \leq 0$ for $t \in[T-\tau, T]$. Since also $w \geq 0$ it follows that $w(t) \equiv 0$ for $t \in[T-2 \tau, T-\tau]$. This process can be continued, and we eventually conclude that $w(t) \equiv 0$ on an interval of values of $t$ such that $t-\tau<0$ for at least some of the $t$. On this interval we will again have 
$b(N(t-\tau ; \lambda)) \equiv \lambda b(N(t-\tau))$ and, for the $t$ such that $t-\tau<0$, this can be rewritten as $b\left(\lambda N_{0}(t-\tau)\right) \equiv \lambda b\left(N_{0}(t-\tau)\right)$ which is only possible if $N_{0}(t-\tau)=0$. However, the verification of strict subhomogeneity only involves $N_{0}$ satisfying $N_{0} \gg 0$. We have therefore shown that $F$ is strictly subhomogeneous. Some of the later stages of this argument will not always be required, it depends on how large $\tau$ is relative to $T$.

Next we shall prove that $F^{A}$ is strongly monotone for some suitably large integer $A$ (depending on $\tau$ and $T$ ). To do so involves showing that $F^{A}$ is monotone and, additionally, that $F^{A}(N) \ll F^{A}(v)$ whenever $N<v$. We shall concentrate on the latter. To see why it is necessary to work with $F^{A}$ instead of $F$, let us first address how we would attempt to prove strong monotonicity of $F$. It would be necessary to show that

$$
\inf _{t \in[T-\tau, T]}[v(t)-N(t)]>0
$$

whenever $N(t)$ and $v(t)$ are the solutions of

$$
\begin{aligned}
& N^{\prime}(t)=e^{-\mu_{j} \tau} b(N(t-\tau))-\mu N(t)-f(t) N(t), \quad N(s)=N_{0}(s), s \in[-\tau, 0], \\
& v^{\prime}(t)=e^{-\mu_{j} \tau} b(v(t-\tau))-\mu v(t)-f(t) v(t), \quad v(s)=v_{0}(s), s \in[-\tau, 0],
\end{aligned}
$$

and $N_{0}$ and $v_{0}$ are such that $N_{0}<v_{0}$, i.e.

$$
N_{0}(s) \leq v_{0}(s) \quad \text { with } \quad N_{0}(s) \not \equiv v_{0}(s) \quad \text { on }[-\tau, 0] .
$$

Letting $y=v-N$, we need to show that $y(t)$ is strictly positive throughout the interval $t \in[T-\tau, T]$. Now, $y(s) \geq 0$ and $y(s) \not \equiv 0$ on $[-\tau, 0]$ and, for $t>0$,

$$
y^{\prime}(t)=y(t-\tau) e^{-\mu_{j} \tau} b^{\prime}(\psi(t))-\mu y(t)-f(t) y(t)
$$

for some function $\psi(t)$ which arises from an application of the mean value theorem. Making use of the method of steps and $b^{\prime}(\cdot)>0$, one easily proves non-negativity of $y(t)$. Strict positivity of $y(t)$ is deduced by making use of $y(s) \not \equiv 0$ on $[-\tau, 0]$, but it is only guaranteed for $t \geq \tau$ (the issues are similar to those in the proof of Proposition 1) and not necessarily for all $t \geq 0$. We need $y(t)>0$ throughout $[T-\tau, T]$, so if $\tau \leq T-\tau$, i.e. $2 \tau \leq T$, then there is no problem and $F$ has been shown to be strongly monotone. If $\tau>T-\tau$ then it is not assured that $y(t)>0$ throughout $[T-\tau, T]$ but we could work with the operator $F^{2}$, which maps the initial datum to the solution on $[2 T-\tau, 2 T]$ rather than $[T-\tau, T]$. Strong monotonicity of $F^{2}$ will be assured if $\tau \leq 2 T-\tau$, i.e. if $\tau \leq T$. The idea in fact is to choose an integer $A$ such that $\tau \leq A T-\tau$, or $2 \tau \leq A T$. One then has strong monotonicity of $F^{A}$.

Thus, $F^{A}$ is the operator that we work with at this stage. A fixed point of $F^{A}$ only assures us of the existence of a periodic solution of (1) of period $A T$ rather than $T$, but this issue will be dealt with later. Let us confirm that $F^{2}$ is strictly subhomogeneous (that the same is true for $F^{A}$ can be shown similarly). It is necessary to show that $F^{2}(\lambda N)>\lambda F^{2}(N)$ when $N \gg 0$ and $\lambda \in(0,1)$. Now, $F(\lambda N)>\lambda F(N)$. The above arguments on monotonicity confirm that $F$ itself is monotone, though not necessarily strongly so. Therefore $F^{2}(\lambda N) \geq F(\lambda F(N))$. It is easily shown that $F(N) \gg 0$ when $N \gg 0$. Therefore, by strict subhomogeneity of $F, F(\lambda F(N))>\lambda F(F(N))$. Hence $F^{2}(\lambda N)>\lambda F^{2}(N)$.

It is also necessary to verify a hypothesis on the Fréchet derivative $D F(0)$ (or $D F^{A}(0)$ if necessary) namely, that it must be compact and strongly positive. This derivative is the linear operator which maps $N_{0}$ to $N_{T}$ (or $N_{A T}$ in the case of $F^{A}$ ) as determined by the linearisation of $(1)$ at its zero solution. To show strong positivity it is necessary to show that $N_{T}$ (or $N_{A T}$ ) is in int $C$ whenever $N_{0}>0$. This has effectively been done already because it amounts to the study of a linear delay 
equation like equation (15) but with $b^{\prime}(0)$ in place of $b^{\prime}(\psi(t))$. The basic issues are the same and are to do with obtaining strict positivity throughout an interval to ensure membership of int $C$. Strong positivity can be shown for $D F^{A}(0)$, with the same $A$ as that required for strong monotonicity.

Finally, one can show the existence of an order interval $V=[0, K] \subset C$ with $F: V \rightarrow V$. Indeed, since $b(\cdot)$ is bounded, we have

$$
N^{\prime}(t) \leq e^{-\mu_{j} \tau} b_{\text {sup }}-\mu N(t)-f_{\min } N(t) .
$$

where $b_{\text {sup }}=\sup _{N \geq 0} b(N)$. Let $\epsilon \in\left(0, \mu+f_{\text {min }}\right)$ and let $N_{0} \in\left[0, e^{-\mu_{j} \tau} b_{\text {sup }} /(\mu+\right.$ $\left.\left.f_{\text {min }}-\epsilon\right)\right]$. We claim that, for all $t>0, N(t) \in\left[0, e^{-\mu_{j} \tau} b_{\text {sup }} /\left(\mu+f_{\text {min }}-\epsilon\right)\right]$ as an interval of $\mathbb{R}$. If $N(t)$ should leave this interval then, at the first time $t_{1}$ of doing so, $N\left(t_{1}\right)=e^{-\mu_{j} \tau} b_{\text {sup }} /\left(\mu+f_{\min }-\epsilon\right)$ and $N^{\prime}\left(t_{1}\right) \geq 0$. But

$$
N^{\prime}\left(t_{1}\right) \leq e^{-\mu_{j} \tau} b_{\text {sup }}-\frac{\left(\mu+f_{\min }\right)}{\left(\mu+f_{\min }-\epsilon\right)} e^{-\mu_{j} \tau} b_{\text {sup }}<0,
$$

a contradiction. Thus $0 \leq N(t) \leq K$ for all $t>0$, where $K=e^{-\mu_{j} \tau} b_{\text {sup }} /(\mu+$ $\left.f_{\min }-\epsilon\right)$, and every positive orbit of $F$ in $[0, K]$ is bounded. Note also that, since this works for any $\epsilon \in\left(0, \mu+f_{\text {min }}\right), K$ can be made large enough to accommodate any given initial data.

We may now apply Theorem 2.3.4 in [27], to the operator $F^{A}$ at this stage. Letting $\rho\left(D F^{A}(0)\right)$ denote the spectral radius of $D F^{A}(0)$, we conclude that if $\rho\left(D F^{A}(0)\right) \leq 1$ then every positive orbit of $F^{A}$ in $V$ converges to 0 , and if $\rho\left(D F^{A}(0)\right)>1$ then there exists a unique fixed point $N^{\bullet} \gg 0$ of $F^{A}$ in $V$ such that every positive orbit of $F^{A}$ in $V \backslash\{0\}$ converges to $N^{\bullet}$. A fixed point of $F^{A}$ corresponds to a periodic solution of (1) of period $A T$. The statements of the theorem follow if we can show that the solution we have found in the case $\rho\left(D F^{A}(0)\right)>1$ has period $T$, as well as $A T$. Note $\rho\left(D F^{A}(0)\right)=\rho\left((D F(0))^{A}\right)=(\rho(D F(0)))^{A}$ so $\rho(D F(0))>1$. Moreover $D F(0)$ is a positive operator, though not necessarily a strongly positive one. Letting $\rho_{*}=\rho(D F(0))$, we find from Theorem 3 that there exists a function $N^{*}>0$ such that $D F(0) N^{*}=\rho_{*} N^{*}$. Next we claim that, in fact, $N^{*} \gg 0$. This follows from the fact that $N^{*}$ will also satisfy $D F^{A}(0) N^{*}=\rho_{*}^{A} N^{*}$ and therefore is a positive eigenvector of $D F^{A}(0)$ corresponding to $\rho_{*}^{A}$. But $D F^{A}(0)$ is a strongly positive operator so the second part of Theorem 3 now applies, with its statement about uniqueness, and informs us that $N^{*} \gg 0$.

From Taylor's theorem in Banach spaces,

$$
F(N)=F(0)+D F(\theta) N
$$

for some $\theta \in X$ that lies on the line segment between 0 and $N$. In our case, and as applied to $\delta N^{*}$ where $\delta>0$ is a small number, this gives

$$
F\left(\delta N^{*}\right)=D F\left(\theta_{\delta}\right) \delta N^{*}
$$

with $\theta_{\delta} \rightarrow 0$ in $X$, as $\delta \rightarrow 0$. Therefore

$$
\begin{aligned}
F\left(\delta N^{*}\right)-\delta N^{*} & =D F\left(\theta_{\delta}\right) \delta N^{*}-\delta N^{*} \\
& =\left(D F\left(\theta_{\delta}\right)-D F(0)\right) \delta N^{*}+D F(0) \delta N^{*}-\delta N^{*} \\
& =\left(D F\left(\theta_{\delta}\right)-D F(0)\right) \delta N^{*}+\left(\rho_{*}-1\right) \delta N^{*}
\end{aligned}
$$

so that

$$
\frac{1}{\delta}\left(F\left(\delta N^{*}\right)-\delta N^{*}\right)=\left(D F\left(\theta_{\delta}\right)-D F(0)\right) N^{*}+\left(\rho_{*}-1\right) N^{*}
$$

Since $\rho_{*}>1$, the right hand side of the above expression is in $\operatorname{int} C$ when $\delta=0$, and therefore remains in int $C$ when $\delta>0$ is sufficiently small, by continuity. Therefore, for sufficiently small $\delta>0, F\left(\delta N^{*}\right) \gg \delta N^{*}$. 
We now define a sequence $N^{(a)} \in X$ by $N^{(0)}=\delta N^{*}$ and $N^{(a+1)}=F\left(N^{(a)}\right)$, $a=0,1,2,3, \ldots$. We have already established that $N^{(1)} \gg N^{(0)}$ and we claim that $N^{(a+1)} \geq N^{(a)}$ for each integer $a \geq 1$. If this is true for a particular $a$ then it is also true for the next $a$, since $N^{(a+2)}=F\left(N^{(a+1)}\right) \geq F\left(N^{(a)}\right)=N^{(a+1)}$ (using that $F$ is monotone) so our claim follows by induction. The monotonicity and boundedness of $N^{(a)}$ in $X$ implies the monotonicity and boundedness of $N^{(a)}(\theta)$ in $\mathbb{R}$, for each fixed $\theta \in[-\tau, 0]$ so that there exists a function $N^{\bullet \bullet} \in X$ with $N^{(a)}(\theta) \rightarrow N^{\bullet \bullet}(\theta)$ as $a \rightarrow \infty$, for each $\theta \in[-\tau, 0]$. Note that $N^{\bullet \bullet}$ is a fixed point of $F$ and therefore has period $T$ (also $N^{(a)}(\theta)=N^{(0)}(a T+\theta)$ for each $\left.a=1,2,3, \ldots\right)$. Moreover the $T$ periodic solution $N^{\bullet \bullet}$ is not the trivial solution since the above monotone iteration starts with $\delta N^{*} \gg 0$. It remains to show that $N^{\bullet \bullet}=N^{\bullet}$, but this is obviously so because any subsequence of $\left\{N^{(a)}\right\}$ must also converge to $N^{\bullet \bullet}$, and so in particular this is true of the subsequence consisting of every $A$ th term. This subsequence is precisely the iterates of $F^{A}$, which converge to $N^{\bullet}$. Thus $N^{\bullet \bullet}=N^{\bullet}$.

Theorem 4 is a threshold result. It is useful in the sense that, under the hypotheses, it offers just the two possibilities of having a globally attractive non-trivial periodic solution, or having the zero solution as a global attractor, and it states clearly the precise circumstances under which each alternative is realised - it depends quite simply on whether the spectral radius of the operator $D F(0)$ is greater than or less than 1, and an important implication of this is that the distinction between the two alternatives can be established by studying the linearised equation about the zero solution, which is what we shall do next. In this way we can obtain sufficient conditions for extinction (or otherwise) that are verifiable in practice, and in so doing we are effectively estimating the spectral radius of $D F(0)$, which cannot be calculated explicitly in terms of the model parameters. We will prove the following theorem.

Theorem 5. Let

$$
Q(t)=e^{-\mu_{j} \tau} b^{\prime}(0) \int_{-\infty}^{t} X(t, z) d z
$$

where

$$
X(t, s)=\exp \left(-\mu(t-s)-\int_{s}^{t} f(\xi) d \xi\right) .
$$

Then $Q(t)$ is periodic of period T. Moreover:

(i) if $\max _{t \in[0, T]} Q(t)<1$ then the spectral radius of $D F(0)$ is less than 1 , and therefore all non-negative solutions $N(t)$ of the linearisation of (1):

$$
N^{\prime}(t)=e^{-\mu_{j} \tau} b^{\prime}(0) N(t-\tau)-\mu N(t)-f(t) N(t)
$$

tend to zero as $t \rightarrow \infty$;

(ii) if $\min _{t \in[0, T]} Q(t)>1$ then the spectral radius of $D F(0)$ is greater than 1 , and therefore the zero solution of (18) is unstable.

Proof. Floquet theory implies that if $\exp (\lambda T)$ is the spectral radius of $D F(0)$ then there exists a $T$-periodic function $p(t)$ such that $N(t)=p(t) \exp (\lambda t)$ satisfies (18). Therefore

$$
p^{\prime}(t)=-\lambda p(t)+e^{-\mu_{j} \tau} b^{\prime}(0) e^{-\lambda \tau} p(t-\tau)-\mu p(t)-f(t) p(t)
$$

Let

$$
X^{\lambda}(t, s)=e^{-\lambda(t-s)} X(t, s)
$$


with $X(t, s)$ given by (17). Note that, since $f(t)$ is periodic of period $T$,

$$
X(t+T, s+T)=X(t, s) .
$$

The periodicity property of $Q(t)$ can be proved easily:

$$
\begin{aligned}
Q(t+T) & =e^{-\mu_{j} \tau} b^{\prime}(0) \int_{-\infty}^{t+T} X(t+T, z) d z \\
& =e^{-\mu_{j} \tau} b^{\prime}(0) \int_{-\infty}^{t} X(t+T, z+T) d z \\
& =e^{-\mu_{j} \tau} b^{\prime}(0) \int_{-\infty}^{t} X(t, z) d z \quad u \operatorname{using}(20) \\
& =Q(t) .
\end{aligned}
$$

Suppose for contradiction that $\max _{t \in[0, T]} Q(t)<1$ but that the spectral radius $\exp (\lambda T)$ of $D F(0)$ is greater than 1 , so that $\lambda>0$. From (19), after some computations, we find that

$$
p(t)=X^{\lambda}(t, 0) p(0)+e^{-\mu_{j} \tau} b^{\prime}(0) e^{-\lambda \tau} \int_{0}^{t} p(z-\tau) X^{\lambda}(t, z) d z .
$$

Let $k$ be any positive integer. Then

$$
p(t+k T)=X^{\lambda}(t+k T, 0) p(0)+e^{-\mu_{j} \tau} b^{\prime}(0) e^{-\lambda \tau} \int_{0}^{t+k T} p(z-\tau) X^{\lambda}(t+k T, z) d z
$$

so that, by periodicity of $p(t)$ and the property $(20)$ of $X(t, s)$,

$$
\begin{aligned}
p(t)= & p(t+k T)=X^{\lambda}(t+k T, 0) p(0) \\
& +e^{-\mu_{j} \tau} b^{\prime}(0) e^{-\lambda \tau} \int_{-k T}^{t} \underbrace{p(k T+z-\tau)}_{=p(z-\tau)} X^{\lambda}(t+k T, z+k T) d z \\
= & X^{\lambda}(t+k T, 0) p(0) \\
& +e^{-\mu_{j} \tau} b^{\prime}(0) e^{-\lambda \tau} \int_{-k T}^{t} p(z-\tau) e^{-\lambda(t-z)} X(t, z) d z .
\end{aligned}
$$

For each fixed $t$ the first term in this expression tends to zero as $k \rightarrow \infty$. Indeed, for $k$ sufficiently large that $t+k T \geq 0$, we have

$$
\begin{aligned}
X^{\lambda}(t+k T, 0) & =e^{-\lambda(t+k T)} X(t+k T, 0) \\
& =e^{-\lambda(t+k T)} \exp \left(-\mu(t+k T)-\int_{0}^{t+k T} f(\xi) d \xi\right) \\
& \leq e^{-\lambda(t+k T)} \rightarrow 0 \quad \text { as } k \rightarrow \infty
\end{aligned}
$$

since we are assuming $\lambda>0$. Letting $k \rightarrow \infty$ in expression (21) and again using that $\lambda>0$, we have

$$
\begin{aligned}
p(t) & \leq e^{-\mu_{j} \tau} b^{\prime}(0) e^{-\lambda \tau} \max _{t \in[0, T]} p(t) \int_{-\infty}^{t} e^{-\lambda(t-z)} X(t, z) d z \\
& \leq e^{-\mu_{j} \tau} b^{\prime}(0) \max _{t \in[0, T]} p(t) \int_{-\infty}^{t} X(t, z) d z
\end{aligned}
$$


giving

$$
p(t) \leq Q(t) \max _{t \in[0, T]} p(t)
$$

for all $t$, with $Q(t)$ defined by (16). But there exists $t^{*}$ with $p\left(t^{*}\right)=\max _{t \in[0, T]} p(t)$ and it follows that $Q\left(t^{*}\right) \geq 1$, which contradicts $\max _{t \in[0, T]} Q(t)<1$. This completes the proof of part (i) of the theorem.

The proof of part (ii) is similar but the details differ slightly near the end. Suppose that $\min _{t \in[0, T]} Q(t)>1$ but that $\lambda<0$. The difference is in how we handle (21), and here we need the fact that $p(t)>0$, which is assured by the Krein-Rutman theorem. Therefore $X^{\lambda}(t+k T, 0) p(0)>0$ and

$$
\begin{aligned}
p(t) & >e^{-\mu_{j} \tau} b^{\prime}(0) e^{-\lambda \tau} \int_{-k T}^{t} p(z-\tau) e^{-\lambda(t-z)} X(t, z) d z \\
& \geq e^{-\mu_{j} \tau} b^{\prime}(0) \int_{-k T}^{t} p(z-\tau) X(t, z) d z \quad \text { since } \lambda<0 .
\end{aligned}
$$

Taking the limit as $k \rightarrow \infty$ and then estimating gives

$$
p(t) \geq Q(t) \min _{t \in[0, T]} p(t)
$$

with $Q(t)$ still given by (16). But there exists $t^{* *}$ at which $p(t)$ attains its minimum, and then $Q\left(t^{* *}\right) \leq 1$, which contradicts $\min _{t \in[0, T]} Q(t)>1$.

Remark 1. It is useful to check Theorem 5 in the particular case when $f(t)$ is a constant, $f(t) \equiv f_{0}>0$, say. In this case we know that the condition for all solutions of (18) to tend to zero is $e^{-\mu_{j} \tau} b^{\prime}(0)<\mu+f_{0}$. In this situation the quantity $Q(t)$ defined by (16) is constant and turns out to be $Q(t) \equiv e^{-\mu_{j} \tau} b^{\prime}(0) /\left(\mu+f_{0}\right)$. If this ratio is less than 1 then part (i) of the theorem applies and we recover the condition $e^{-\mu_{j} \tau} b^{\prime}(0)<\mu+f_{0}$ that we expect in this autonomous case.

Remark 2. Theorem 5 only tells us that the long time average is reduced by culling if condition (i) holds (in the statement of the theorem) and $e^{-\mu_{j} \tau} b^{\prime}(0) \geq \mu$, since if $e^{-\mu_{j} \tau} b^{\prime}(0)<\mu$ then $N(t) \rightarrow 0$ as $t \rightarrow \infty$ in the absence of culling by Theorem 1 in [13].

Our next result concerns the possibilities that $b(N)$ is increasing for all $N$, and the possibility that $b(N)$ satisfies (5) in the case that $N^{*}<N_{m}$ (i.e. $N^{*}$ is on the "increasing" side of $b(N))$.

Theorem 6. In model (1) suppose that $f(t) \geq 0$. Then culling lowers the long time mean of the population in either of the following sets of circumstances:

(i) $b(0)=0$ and $b(N)$ is non-decreasing for all $N>0$;

(ii) $b(N)$ satisfies (5) with $N^{*}<N_{m}$.

Proof. We start with case (i). Even though we only have $b(N) \geq 0$ (and not $>0$ ) under the hypotheses of case (i), non-negativity of $N(t)$ still holds (Smith [23], p81). Thus, since $f(t) \geq 0$,

$$
N^{\prime}(t) \leq e^{-\mu_{j} \tau} b(N(t-\tau))-\mu N(t) .
$$

Since $b(N)$ is non-decreasing, Theorem 5.1.1 on page 78 of Smith [23] applies and enables us to state that $N(t) \leq \tilde{N}(t)$ where

$$
\tilde{N}^{\prime}(t)=e^{-\mu_{j} \tau} b(\tilde{N}(t-\tau))-\mu \tilde{N}(t), \quad \tilde{N}(s)=N_{0}(s) \geq 0, s \in[-\tau, 0]
$$


so that the long time average of $N(t)$ is bounded by the long time average in the absence of culling (further information would be required to determine the actual dynamics of solutions of (22), but relevant results are known and can be found, for example, in Kuang [28]).

In case (ii) we may state that

$$
\begin{aligned}
N^{\prime}(t) & \leq e^{-\mu_{j} \tau} b(N(t-\tau))-\mu N(t) \\
& \leq e^{-\mu_{j} \tau} b\left(N_{m}\right)-\mu N(t)
\end{aligned}
$$

so that

$$
\limsup _{t \rightarrow \infty} N(t) \leq \frac{e^{-\mu_{j} \tau} b\left(N_{m}\right)}{\mu}
$$

It is easy to show that

$$
N^{*}<\frac{e^{-\mu_{j} \tau} b\left(N_{m}\right)}{\mu}<N_{m}
$$

Therefore there exists $t_{0}$ such that, for all $t \geq t_{0}, N(t) \leq N_{m}$. Then for all times $t \geq t_{0}+\tau$ there is no longer any history of the solution $N(t)$ having ever exceeded $N_{m}$. If we translate time so that $t_{0}+\tau$ is the new initial time, and $\left\{N(t): t \in\left[t_{0}, t_{0}+\tau\right]\right\}$ the initial data, then for the new initial value problem $b(N)$ is effectively increasing for all $N$ and so we are back in case (i). Note that the initial transients (the values of $N$ on the interval $\left[0, t_{0}+\tau\right]$ ) cannot contribute to the long time average.

In this and the previous section we have discovered circumstances under which culling reduces the mean population. We now turn our attention to constructing circumstances under which it increases the mean.

\section{The role of the birth function}

Recall figure 1 in section 3 showing that infrequent culling can maintain the average pest population at an unnaturally high level. In fact, figure 1 shows that, without culling, the pest population tends to a positive constant and that, when subject to the particular culling regime chosen for the simulation, the population exhibits steady oscillations and tends to a periodic solution where the period equals the inter-cull time. Moreover the mean value for this periodic solution, taken over one such inter-cull period, clearly exceeds the constant to which the population tends in the absence of culling.

Let us now try to account for this behaviour in terms of the shape of the birth function. The birth function used in figure 1 is of Nicholson-type. Thus it is of the form $b(N)=\lambda_{1} N e^{-\lambda_{2} N}$ for $\lambda_{1}, \lambda_{2}$ positive constants. Such a function increases exponentially for small $N$, before levelling off due to the exponential factor, and finally decreasing, tending to zero as $N \rightarrow \infty$ - see figure 2 (right). We can say that a Nicholson birth function $b(N)$ is biggest when the population $N$ is small or intermediate, whereas $b(N)$ is small if $N$ is very small or if $N$ is large.

Consider what happens in the absence of culling if the initial data $N(t)=N_{0}(t)$ for $t \in[-\tau, 0]$ is in the intermediate range of $N$ for which $b(N)$ is large. One can envisage that the population will grow on the interval $t \in[0, \tau]$, provided the death rate $\mu$ is not too high, because $b(N(t-\tau))$ will be large in (1). The population, then, will be higher for $t \in[0, \tau]$ than it was initially. For the next time interval $t \in[\tau, 2 \tau]$, the term $b(N(t-\tau))$ may now be smaller, because $N(t-\tau)$ will have grown and $b(N)$ becomes small for large $N$. Suppose $N(t-\tau)$ has become large enough that $b(N(t-\tau))$ is small. Then by (1) we realise that $N(t)$ will decrease. 
Indeed it may decrease to be around the same level it was for the initial data. If this is so, then the pattern just described may repeat indefinitely (stable oscillations have been observed in the model (1), as mentioned in [20]) or the oscillations may die down as the population tends to a fixed point (see figure 1 and corollary 9.3 on page 163 of [28]).

It is the discrepancy in the size of the birth function $b(N)$ for intermediate $N$ and large $N$, as well as the delayed response of the system in (1), that permit oscillations to occur at all. In the absence of delay, $N(t)$ would tend monotonically to a fixed point.

Now consider what happens when a culling regime is imposed in the situation where, in the absence of culling, $N(t)$ tends to a fixed point. Imagine as before that the initial data is in the intermediate range of $N$ for which $b(N)$ is large. We have seen how this may cause $b(N)$ to then increase before eventually decreasing again. Suppose we impulsively cull the population whilst it is still above the intermediate range of $N$. We choose a cull strength which reduces $N(t)$ to be a little bigger than the $N$ for which $b(N)$ is maximum. How $N(t)$ then behaves will depend upon $N(t-\tau)$. But recently (if "now" is the time of the cull) the population was above the intermediate range of $N$ for which $b(N)$ is large. Conceivably, then, $N(t-\tau)$ is large enough that $b(N(t-\tau))$ will not be large. Then, by $(1), N(t)$ may decrease across the range of intermediate $N$. If $N(t)$ has not decreased below the range of intermediate $N$ before $\tau$ time units has elapsed, then we may have recovered the initial data, which was chosen to be in the intermediate range of $N$. The pattern just described may now repeat.

Thus, strategically chosen culling may create a periodic solution when, in the absence of culling, the population would tend to a fixed point. If $b(N)$ is large enough for intermediate $N$, then initial data (for $t \in[-\tau, 0]$ ) in this intermediate range of $N$ will cause $N(t)$ to increase quite significantly when $t \in[0, \tau]$. We envisage a scenario in which $b(N)$ is sufficiently large for intermediate $N$ such that if culling does create a periodic solution then the average value of $N(t)$ over one period is larger than the fixed point to which $N(t)$ tends in the absence of culling. By creating this scenario, we have established a mechanism to explain the features revealed by figure 1 .

\section{$7 \quad$ A simple birth function}

Motivated by the previous section, we note that a birth function can be large for intermediate $N$ and smaller for larger $N$ if it satisfies:

$$
b(N)= \begin{cases}W & \text { for } N_{1} \leq N<N_{2}, \\ M & \text { for } N_{2} \leq N \leq N_{3},\end{cases}
$$

where $N_{1}, N_{2}, N_{3}, M$, and $W$ are positive constants with $N_{1}<N_{2}<N_{3}$ and $W>M$. The range $N \in\left[N_{1}, N_{2}\right)$ is to be considered "intermediate" and $N \in$ $\left[N_{2}, N_{3}\right]$ is "larger". The behaviour of the birth function $b(N)$ for $N<N_{1}$ and $N>N_{3}$ will not influence our results, so we will allow $b(N)$ to be any plausible birth function for these ranges of $N$. In particular it is plausible to ask for $b(N)$ to increase monotonically in some way from zero to $W$ for $N \in\left[0, N_{1}\right]$ and for $b(N)$ to decrease monotonically from $M$ to zero for $N \geq N_{3}$

How realistic is it to require a birth function $b(N)$ to satisfy $(23)$ ? The idea that $b(N)$ is roughly constant for intermediate $N$ is relatively sensible. After all, we can expect $b(N)$ to increase with $N$ for small $N\left(0<N<N_{1}\right)$ until competition for food, space, or a successful mating prevent the birth rate from rising any further. The birth rate may not fall immediately, however, because if the population continues to grow then there will be more individuals who can reproduce even though 

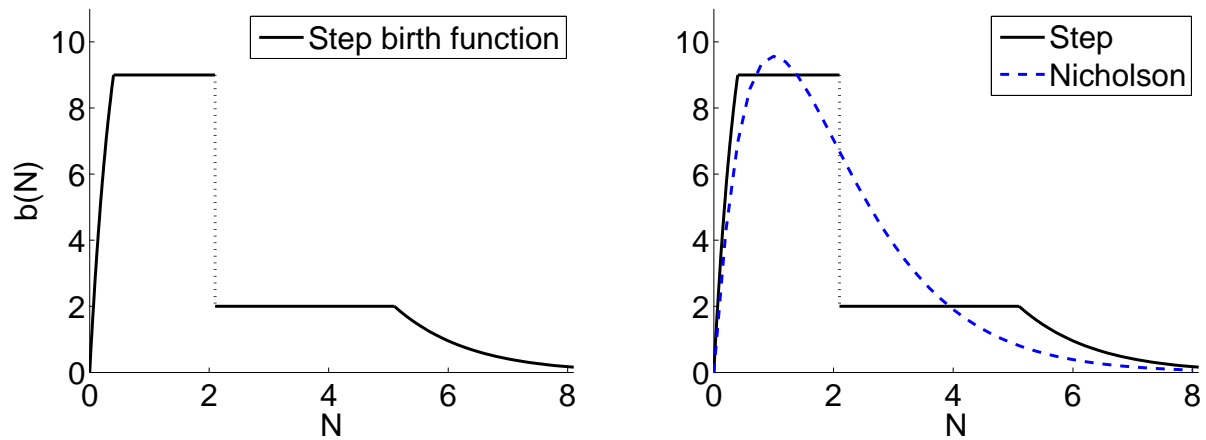

Figure 2: Left: a step birth function. Right: Comparison of a step birth function and a Nicholson birth function. For step birth function in both left and right plots: $N_{1}=0.4, N_{2}=2.1, N_{3}=5.1, M=2, W=9, b(N)=\lambda_{1} N e^{-\lambda_{2} N}$ on $\left[0, N_{1}\right]$ where $\lambda_{2}=1$ and $\lambda_{1}=\frac{W}{N_{1}} e^{\lambda_{2} N_{1}}$, and $b(N)=\lambda_{1} N e^{-\lambda_{2} N}$ on $\left[N_{3}, \infty\right)$ where $\lambda_{2}=1$ and $\lambda_{1}=\frac{M}{N_{3}} e^{\lambda_{2} N_{3}}$. Nicholson birth function: $b(N)=26 N e^{-N}$.

competition will intensify. These two opposing influences - a growing population and growing competition - may balance each other (thereby yielding a flat birth function across intermediate $N$ ) until the competition and other environmental pressures are sufficiently serious as to trigger a sudden collapse in the birth rate. After the birth rate $b(N)$ collapses where $N=N_{2}$, say, from some value $W$ to some value $M$, it may remain at roughly $M$ for a range of $N>N_{2}$ because the two opposing forces - a growing population and growing competition or environmental pressures - may once again balance. Otherwise $b(N)$ may decrease to zero for $N>N_{2}$, but if it does this slowly enough at first then $b(N)$ may be approximated by a constant for a range of $N>N_{2}$.

A birth function satisfying (23) can be called a step birth function. The appropriateness of such a label is made obvious by plotting such a function, which we do in figure 2 (left). In figure 2 (right) we compare a step birth function with a Nicholson birth function. The two functions are qualitatively not dissimilar.

\section{Model analysis}

The following assumptions will be used in this section, though they will not all be assumed to hold simultaneously:

(A1) The birth function $b(N)$ satisfies (23) for $N_{1} \leq N \leq N_{3}$; also $b(N)$ is monotonic increasing for $0<N<N_{1}$ and monotonic decreasing for $N>N_{3}$;

(A2) $N(t)$ satisfies $d N(t) / d t=e^{-\mu_{j} \tau} b(N(t-\tau))-\mu N(t)$ for $t>0$;

(A3) $N(t)$ satisfies $d N(t) / d t=e^{-\mu_{j} \tau} b(N(t-\tau))-\mu N(t)$ for $t>0$ except at times $t_{i}$ $(i \geq 1)$ where $N(t)$ is impulsively rescaled according to $N\left(t_{i}\right)=\left(1-b_{i}\right) N\left(t_{i}^{-}\right)$ where $t_{i}^{-}$is the time momentarily before $t_{i}$ and $0 \leq b_{i} \leq 1$. We think of $t_{i}$ as the time of the $i$-th cull and $b_{i}$ as the strength of the $i$-th cull.

Notice that assumption (A2) is the same as equation (1) with $f(t) \equiv 0$, so that the population is not subject to culling. Notice also that assumption (A3) is the same as equation (1) with $f(t)$ satisfying (4). 


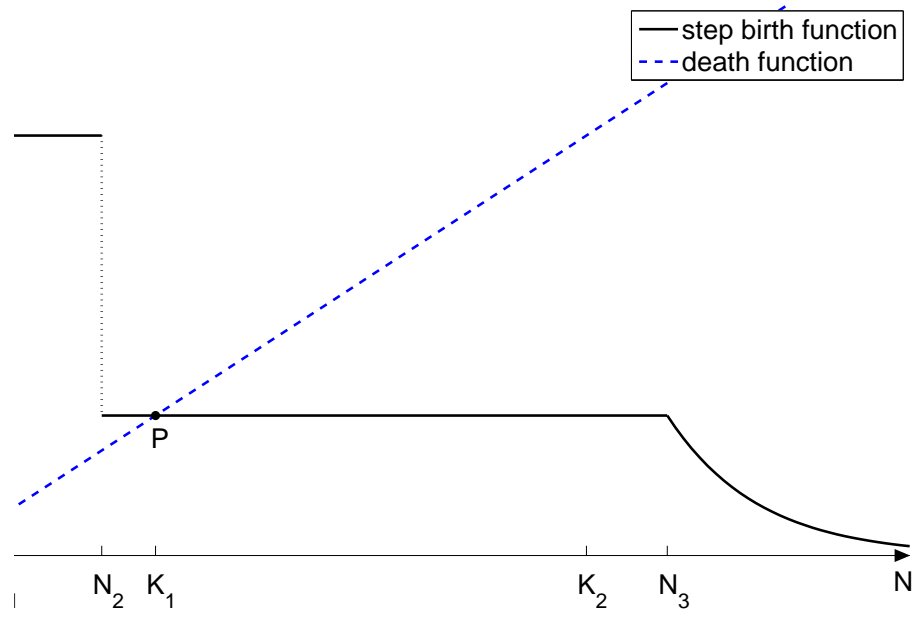

Figure 3: Step birth function and death function - picture to accompany theorem 7 . For step birth function: $N_{1}=0.7, N_{2}=2, N_{3}=9, M=0.8, W=2.4, b(N)=$ $\lambda_{1} N e^{-\lambda_{2} N}$ on $\left[0, N_{1}\right]$ where $\lambda_{2}=1$ and $\lambda_{1}=\frac{W}{N_{1}} e^{\lambda_{2} N_{1}}$, and $b(N)=\lambda_{1} N e^{-\lambda_{2} N}$ on $\left[N_{3}, \infty\right)$ where $\lambda_{2}=1$ and $\lambda_{1}=\frac{M}{N_{3}} e^{\lambda_{2} N_{3}}$. Death function is $\mu N$ where $\mu=0.3$. Here $K_{1}=e^{-\mu_{j} \tau} \frac{M}{\mu}$ and $K_{2}=e^{-\mu_{j} \tau} \frac{W}{\mu}$. The birth and death functions intersect at $\mathbf{P}$, where $N=K_{1}=e^{-\mu_{j} \tau} \frac{M}{\mu}$.

In this section we will show that $N(t)$ can tend to a fixed point $N^{*}$ in the absence of culls and that a culling regime may be chosen which forces $N(t)$ to be periodic where the periodic solution will be stated explicitly.

Theorem 7. Assume (A1) and (A2) hold. Suppose $\mu$ satisfies $N_{2}<e^{-\mu_{j} \tau} \frac{M}{\mu}<$ $e^{-\mu_{j} \tau} \frac{W}{\mu}<N_{3}$. Also suppose $N(t) \in\left[N_{1}, N_{3}\right]$ for $t \in[-\tau, 0]$. Then $N(t) \rightarrow$ $e^{-\mu_{j} \tau} \frac{M}{\mu}=N^{*}$ as $t \rightarrow \infty$.

Proof. Figure 3 depicts the situation.

Using the initial data, there will always be two options for $t \in[0, \tau]$ :

(i) $d N(t) / d t=e^{-\mu_{j} \tau} W-\mu N(t)$, in which case $N(t)$ moves monotonically towards $e^{-\mu_{j} \tau} W / \mu$

(ii) $d N(t) / d t=e^{-\mu_{j} \tau} M-\mu N(t)$, in which case $N(t)$ moves monotonically towards $e^{-\mu_{j} \tau} M / \mu$.

Since $N_{2}<e^{-\mu_{j} \tau} \frac{M}{\mu}<e^{-\mu_{j} \tau} \frac{W}{\mu}<N_{3}$, it follows that $N(t) \in\left[N_{1}, N_{3}\right]$ for $t \in[0, \tau]$. It then follows by a method of steps induction that $N(t) \in\left[N_{1}, N_{3}\right]$ for all $t>0$. Hence for any time $t>0$, either option (i) or option (ii) will hold. Since $N_{2}<$ $e^{-\mu_{j} \tau} \frac{M}{\mu}<e^{-\mu_{j} \tau} \frac{W}{\mu}<N_{3}$, we must then have $N(t)>N_{2}$ for all $t$ large enough, say $t \geq T$. But then, for all $t \geq T+\tau$ we will have $b(N(t-\tau))=M$ and option (ii) will hold. Hence $N(t) \rightarrow e^{-\mu_{j} \tau} \frac{M}{\mu}$ as $t \rightarrow \infty$.

Observe that if the initial data in Theorem 7 were defined on the interval $t \in$ $[A, A+\tau]$ for any constant $A \geq-\tau$, instead of on $[-\tau, 0]$, then it is trivial to see that the result would still hold, that is, we would still have $N(t) \rightarrow e^{-\mu_{j} \tau} M / \mu$ as 
$t \rightarrow \infty$. We make this remark in view of the way we define the initial data in the next theorem. Another remark on initial data is made directly after the statement of the next theorem.

Theorem 8. Assume (A1) and (A3) hold. Also assume:

(B1) $N_{1} \leq N(0)<N_{2}<e^{-\mu_{j} \tau} \frac{M}{\mu}<e^{-\mu_{j} \tau} \frac{W}{\mu}<N_{3}$,

(B2) $e^{-\mu_{j} \tau} \frac{M}{\mu}+\left(N(0)-e^{-\mu_{j} \tau} \frac{M}{\mu}\right) e^{-\mu \tau}=N_{2}$.

Then, on $[0,2 \tau)$, provided no culls occur on this interval,

$$
\begin{aligned}
& N(t)=e^{-\mu_{j} \tau} \frac{M}{\mu}+\left(N(0)-e^{-\mu_{j} \tau} \frac{M}{\mu}\right) e^{-\mu t} \text { for } t \in[0, \tau] \text { (initial data), } \\
& N(t)=e^{-\mu_{j} \tau} \frac{W}{\mu}+\left(N_{2}-e^{-\mu_{j} \tau} \frac{W}{\mu}\right) e^{-\mu(t-\tau)} \text { for } t \in[\tau, 2 \tau) .
\end{aligned}
$$

If culls occur every $2 \tau$ time units, with the first cull occurring at time $t_{1}=2 \tau$, and where culls have strength

$$
\delta=1-\frac{N(0)}{e^{-\mu_{j} \tau \frac{W}{\mu}}+\left(N_{2}-e^{-\mu_{j} \tau} \frac{W}{\mu}\right) e^{-\mu \tau}},
$$

then (24) and (25) repeats as a periodic solution for all subsequent time.

We will prove Theorem 8 after commenting on the initial data. Notice, then, that the initial data in Theorem 8 is defined on the interval $t \in[0, \tau]$ as opposed to $[-\tau, 0]$, which is the interval on which the initial data is defined in (2) and in Theorem 7. It is not necessary to define initial data on $[-\tau, 0]$. It is simply a convention. We have defined the initial data in Theorem 8 on the interval $[0, \tau]$ because it makes the notation slightly more elegant in Theorem 8 and the analysis that follows it. If we were to translate the time variable with the transformation $t \rightarrow t-\tau$, then the initial data in Theorem 8 would be defined on the interval $t \in[-\tau, 0]$, and other details in Theorem 8 would also be slightly different. But the method of proof would be essentially the same.

Proof. First note that continuity of the function in (24) and (25) is assured by assumption (B2). Notice also that $0<\delta<1$ by using assumption (B2) and the fact that $N(0)<N_{2}<e^{-\mu_{j} \tau} \frac{W}{\mu}$ by assumption (B1). To prove that (24) and (25) satisfy $d N(t) / d t=e^{-\mu_{j} \tau} b(N(t-\tau))-\mu N(t)$ with $b(N)$ defined by assumption (A1), we use (24) as initial data on $[0, \tau]$ and we shall deduce that (25) holds.

Now by (24) and assumption (B1) we know that $N(0) \geq N_{1}$ and that $N(t)$ is increasing on $[0, \tau]$ since $N(0)-e^{-\mu_{j} \tau} \frac{M}{\mu}<0$. Also by (24) and assumption (B2), we know that $N(\tau)=N_{2}$. Thus we have $N_{1} \leq N(t) \leq N_{2}$ for $t \in[0, \tau]$, so that $N_{1} \leq N(t-\tau) \leq N_{2}$ for $t \in[\tau, 2 \tau]$. But then, by assumption (A1), $b(N(t-\tau))=W$ for $t \in[\tau, 2 \tau]$. Therefore, using the knowledge that $d N(t) / d t=$ $e^{-\mu_{j} \tau} b(N(t-\tau))-\mu N(t)$ for $t \in(\tau, 2 \tau)$ by assumption (A3), we can write

$$
N(\tau)=N_{2} \quad \text { and } \quad \frac{d N(t)}{d t}=e^{-\mu_{j} \tau} W-\mu N(t) \text { for } t \in(\tau, 2 \tau) .
$$

Solving (27) yields (25).

To show that the culling regime stated in the theorem forces (24) and (25) to be periodic, we need only show that the expression in (24), with $t$ replaced by $t-2 \tau$, will hold for $t \in[2 \tau, 3 \tau]$. Then the argument in the first part of the proof will 
ensure the expression in (25), with $t$ replaced by $t-2 \tau$, holds for $t \in[3 \tau, 4 \tau)$, and the periodicity will be guaranteed by induction.

Now we have seen that (25) holds. But then $N(t)$ is increasing on $[\tau, 2 \tau)$ since, by assumption (B1), we have $N_{2}-e^{-\mu_{j} \tau} \frac{W}{\mu}<0$. Hence:

$$
N(\tau)=N_{2} \leq N(t) \leq e^{-\mu_{j} \tau} \frac{W}{\mu}+\left(N_{2}-e^{-\mu_{j} \tau} \frac{W}{\mu}\right) e^{-\mu \tau} \text { for } t \in[\tau, 2 \tau) .
$$

Using assumption (B1), $e^{-\mu_{j} \tau} \frac{W}{\mu}+\left(N_{2}-e^{-\mu_{j} \tau} \frac{W}{\mu}\right) e^{-\mu \tau}<N_{3}$, so by (28) and assumption (A1), we can say that $b(N(t-\tau))=M$ for $t \in[2 \tau, 3 \tau)$. Then, using assumption (A3), we have:

$$
\frac{d N(t)}{d t}=e^{-\mu_{j} \tau} M-\mu N(t) \text { for } t \in(2 \tau, 3 \tau) .
$$

We also know by (25) that $N\left(2 \tau^{-}\right)=e^{-\mu_{j} \tau} \frac{W}{\mu}+\left(N_{2}-e^{-\mu_{j} \tau} \frac{W}{\mu}\right) e^{-\mu \tau}$ where $2 \tau^{-}$ is the time "momentarily" before $2 \tau$. But then by assumption (A3) and (26) we can write $N(2 \tau)=(1-\delta) N\left(2 \tau^{-}\right)=N(0)$. Hence to find $N(t)$ on $[2 \tau, 3 \tau)$, we solve (29) with the initial condition $N(2 \tau)=N(0)$ to obtain the expression in (24), with $t$ replaced by $t-2 \tau$, on the interval [ $2 \tau, 3 \tau)$. Finally there is no cull at $t=3 \tau$ and this guarantees continuity of $N(t)$ at $t=3 \tau$, so the expression in (24), with $t$ replaced by $t-2 \tau$, is obtained on the interval $[2 \tau, 3 \tau]$. The proof is complete.

\section{Influence of culling on long-term mean popula- tion}

The inter-cull average value of the periodic solution in theorem 8 will depend upon $\tau$. In fact, if we denote the inter-cull average by $N^{*}(\tau)$, then:

$$
\begin{aligned}
N^{*}(\tau)= & \frac{1}{2 \tau} \int_{0}^{2 \tau} N(t) d t=\frac{1}{2 \tau}\left\{\int_{0}^{\tau} N(t) d t+\int_{\tau}^{2 \tau} N(t) d t\right\} \\
= & \frac{1}{2} e^{-\mu_{j} \tau}\left(\frac{M}{\mu}+\frac{W}{\mu}\right) \\
& \quad+\frac{1}{2}\left(\frac{1-e^{-\mu \tau}}{\mu \tau}\right)\left(N(0)+N_{2}-e^{-\mu_{j} \tau}\left(\frac{M}{\mu}+\frac{W}{\mu}\right)\right) .
\end{aligned}
$$

Notice that the inter-cull average value of the periodic solution is equal to the longterm average value, namely $\lim _{t \rightarrow \infty}(1 / t) \int_{0}^{t} N(s) d s$. Notice also that $N(0)$ and $N_{2}$ in (30) are related by a condition involving $\tau$, namely condition (B2) in Theorem 8.

Recall by Theorem 7 that the fixed point $e^{-\mu_{j} \tau} \frac{M}{\mu}$ is globally asymptotically stable in the absence of culling, where "global" is understood to mean the region to which the initial data is restricted in the theorem. Therefore, to gain insight into how culling will influence the long-term mean pest population, let us compare the fixed point $e^{-\mu_{j} \tau} \frac{M}{\mu}$ to the average value of the periodic solution in the model with culling, as derived in Theorem 8, namely $N^{*}(\tau)$ in (30). The influence of the culling regime will clearly depend upon the sign of $N^{*}(\tau)-e^{-\mu_{j} \tau} \frac{M}{\mu}$ or, equivalently, on the sign of $e^{\mu_{j} \tau} N^{*}(\tau)-\frac{M}{\mu}$. To be more specific, if we define the function

$$
h(\tau)=e^{\mu_{j} \tau} N^{*}(\tau)-\frac{M}{\mu},
$$


then the culling regime of Theorem 8 increases the long-term mean population if $h(\tau)>0$ and decreases it if $h(\tau)<0$. Since it is meaningless to discuss the influence of the culling regime of Theorem 8 unless that theorem can actually hold, our problem is to investigate the behaviour of the function $h(\tau)$ in $(31)$ when conditions (B1) and (B2) of Theorem 8 hold.

We construct a framework in which conditions (B1) and (B2) of Theorem 8 will hold, and in which a simple investigation of $h(\tau)$ in $(31)$ is possible, by first assuming that $N_{2}, N_{3}, \mu, M$, and $W$ are positive constants satisfying:

$$
N_{2}<\frac{M}{\mu}<\frac{W}{\mu}<N_{3}
$$

We also assume that $\mu_{j}$ is a positive constant.

Next define

$$
\tau_{1}=\frac{1}{\mu_{j}} \ln \left(\frac{\left(\frac{M}{\mu}\right)}{N_{2}}\right) .
$$

Note that $\tau_{1}>0$ because $\frac{M}{\mu}>N_{2}$ by (32). We may rearrange (33) to obtain

$$
N_{2}=e^{-\mu_{j} \tau_{1}}\left(\frac{M}{\mu}\right)
$$

By (32) and (34), we notice that

$$
N_{2}<e^{-\mu_{j} \tau} \frac{M}{\mu}<e^{-\mu_{j} \tau} \frac{W}{\mu}<N_{3} \quad \text { for } \tau<\tau_{1} .
$$

Now define $N(0)$ to be a function of $\tau$ as follows:

$$
N(0)=N(0)_{\tau}=e^{-\mu_{j} \tau} \frac{M}{\mu}+e^{\mu \tau}\left(N_{2}-e^{-\mu_{j} \tau} \frac{M}{\mu}\right) .
$$

Note that (36) is simply a rearrangement of condition (B2) of Theorem 8 , so by defining $N(0)$ as in (36) we trivially allow condition (B2) to hold. The dependence of $N(0)$ on $\tau$ is encapsulated by the notation $N(0)_{\tau}$.

By (35) we know that $N_{2}-e^{-\mu_{j} \tau} \frac{M}{\mu}<0$ for $\tau \in\left(0, \tau_{1}\right)$, so it is not obvious if $N(0)_{\tau}>0$ for every value of $\tau$ such that $\tau \in\left(0, \tau_{1}\right)$. However, if we notice that $N(0)_{\tau}$ is a continuous function of $\tau$ and that $N(0)_{0}=N_{2}>0$, then we may deduce that there exists $\bar{\tau}$ satisfying $0<\bar{\tau}<\tau_{1}$ such that $N(0)_{\tau}>0$ for $\tau \in(0, \bar{\tau})$. If we notice also that $N(0)_{\tau_{1}}=e^{-\mu_{j} \tau_{1}} \frac{M}{\mu}>0$ (using (34)), then we may further deduce that there exists $\hat{\tau}$ satisfying $\bar{\tau} \leq \hat{\tau}<\tau_{1}$ such that $N(0)_{\tau}>0$ for $\tau \in\left(\hat{\tau}, \tau_{1}\right)$. We can summarise the results of this paragraph as follows:

$$
N(0)_{\tau}>0 \quad \text { for } \tau \in(0, \bar{\tau}) \cup\left(\hat{\tau}, \tau_{1}\right) \text { where } 0<\bar{\tau} \leq \hat{\tau}<\tau_{1}
$$

Given that $\tau>0$, routine manipulations allow us to deduce, by (34) and (36), that $N(0)_{\tau}<N_{2}$ if and only if $\tau<\tau_{1}$. We know by (37) that $N(0)_{\tau}>0$ for $\tau \in(0, \bar{\tau}) \cup\left(\hat{\tau}, \tau_{1}\right)$. For any particular $\tau$ such that $\tau \in(0, \bar{\tau}) \cup\left(\hat{\tau}, \tau_{1}\right)$, we can define $N_{1}$ to be any positive constant such that $N_{1}<N(0)_{\tau}$. Combining the observations of the last two sentences with (35), we find that:

$$
N_{1}<N(0)=N(0)_{\tau}<N_{2}<e^{-\mu_{j} \tau} \frac{M}{\mu}<e^{-\mu_{j} \tau} \frac{W}{\mu}<N_{3} \quad \text { for } \tau \in(0, \bar{\tau}) \cup\left(\hat{\tau}, \tau_{1}\right) .
$$

Hence condition (B1) of Theorem 8 holds for $\tau \in(0, \bar{\tau}) \cup\left(\hat{\tau}, \tau_{1}\right)$. 
Knowing that conditions (B1) and (B2) of Theorem 8 hold for $\tau \in(0, \bar{\tau}) \cup\left(\hat{\tau}, \tau_{1}\right)$, we can now investigate the behaviour of the function $h(\tau)$ (defined in (31)) for $\tau \in\left(0, \tau_{1}\right)$. Firstly, then, notice that we can write out an explicit expression for $h(\tau)$ as follows:

$$
\begin{aligned}
& h(\tau)= e^{\mu_{j} \tau} N^{*}(\tau)-\frac{M}{\mu} \quad(\operatorname{using}(31)) \\
&=\left(\frac{W}{\mu}-\frac{M}{\mu}\right)+\left(\frac{1-e^{-\mu \tau}}{\mu \tau}\right)\left[e^{\mu_{j} \tau}\left(N(0)+N_{2}\right)-\left(\frac{M}{\mu}+\frac{W}{\mu}\right)\right] \\
& \quad\left(\frac{W}{\mu}-\frac{M}{\mu}\right)+\left(\frac{1-e^{-\mu \tau}}{\mu \tau}\right)\left[e^{\mu \tau}\left(e^{\mu_{j} \tau} N_{2}-\frac{M}{\mu}\right)+e^{\mu_{j} \tau} N_{2}-\frac{W}{\mu}\right] \\
& \quad(\operatorname{using}(36)) .
\end{aligned}
$$

By L'Hôpital's rule we may deduce that $\lim _{\tau \rightarrow 0}\left(\frac{1-e^{-\mu \tau}}{\mu \tau}\right)=1$. Hence we find by (39) that $\lim _{\tau \rightarrow 0} h(\tau)=2\left(N_{2}-\frac{M}{\mu}\right)$. But $N_{2}-\frac{M}{\mu}<0$ by (32). Therefore:

$$
\lim _{\tau \rightarrow 0} h(\tau)<0
$$

Moreover we may deduce by (34) and (39) that

$$
h\left(\tau_{1}\right)=\left(\frac{W}{\mu}-\frac{M}{\mu}\right)\left[1-\left(\frac{1-e^{-\mu \tau_{1}}}{\mu \tau_{1}}\right)\right] .
$$

It is trivial to check that $\frac{1-e^{-\mu \tau}}{\mu \tau}<1$ for $\tau>0$. In particular, then, $\frac{1-e^{-\mu \tau_{1}}}{\mu \tau_{1}}<1$. Also $\frac{W}{\mu}-\frac{M}{\mu}>0$ by (32). Therefore by (41) we have:

$$
h\left(\tau_{1}\right)>0 .
$$

Since $h(\tau)$ is a continuous function of $\tau$, we may deduce by (40) that there exists $\tau^{*}$ satisfying $0<\tau^{*} \leq \bar{\tau}$ such that

$$
h(\tau)<0 \quad \text { for } \tau \in\left(0, \tau^{*}\right) .
$$

Again, since $h(\tau)$ is continuous in $\tau$, we deduce by (42) that there exists $\tau^{* *}$ satisfying $\hat{\tau} \leq \tau^{* *}<\tau_{1}$ such that

$$
h(\tau)>0 \quad \text { for } \tau \in\left(\tau^{* *}, \tau_{1}\right) .
$$

Using the fact that $\bar{\tau} \leq \hat{\tau}$ by (37) and also using inequalities stated in the last few paragraphs, it is clear that $0<\tau^{*} \leq \tau^{* *}<\tau_{1}$.

We are now in a position to draw some conclusions about the influence of the culling regime of Theorem 8 on the mean pest population. Recalling from the second paragraph of this section that the mean population is decreased when $h(\tau)<0$, we see by (43) that the culling regime decreases the mean population for all $\tau$ sufficiently small. Notice that this is not an obvious result. Although the culling frequency is high when $\tau$ is small (since culls occur every $2 \tau$ time units in Theorem 8), the cull strengths are also weak when $\tau$ is small (since $\delta \rightarrow 1$ as $\tau \rightarrow 0$ where $\delta$ is defined in (26) and where we use the fact that $N(0)=N(0)_{\tau} \rightarrow N_{2}$ as $\tau \rightarrow 0$ where $N(0)$ is defined in (36) or condition (B2) of Theorem 8).

Now recalling from the second paragraph of this section that the mean population is increased when $h(\tau)>0$, we see by (44) that there is a finite range of $\tau$ such that the culling regime of Theorem 8 increases the mean population. 
The analysis of this section has shown that the impact of the culling regime of Theorem 8 on the mean pest population depends upon the size of the delay $\tau$. The mean population is decreased when $\tau$ is suitably small and increased when $\tau$ belongs to a larger - but still finite - range of values. It would clearly be practical if we could explain this dependence on $\tau$ in biological terms but no immediate explanation presents itself to the authors. This issue warrants further research, which could begin with an extensive numerical study involving various forms of unimodal birth function.

\section{Simulations}

We can illustrate by simulation the points made in the previous section. We first choose $N_{2}, N_{3}, \mu, M$, and $W$ to be positive constants satisfying (32), specifically $N_{2}=1.5, M=2, W=10, \mu=1$, and $N_{3}$ can be anything bigger than $\frac{W}{\mu}=10$ and need not be specificied more precisely for the purposes of carrying out our simulations.

As in section 9 , we choose $\mu_{j}$ to be a positive constant. We let $\mu_{j}=0.1$. Notice that we have chosen the juvenile per capita death rate $\mu_{j}$ to be less than the adult per capita death rate $\mu$ to reflect the idea that adults may be driven into the open by the instinct to search for a mate, making them more vulnerable to predation. Given our choices for $N_{2}, M, \mu$, and $\mu_{j}$, we find by (33) that $\tau_{1}=2.8768$.

For any particular $\tau \in\left(0, \tau_{1}\right)$, we define $N(0)=N(0)_{\tau}$ as in (36), so that condition (B2) of Theorem 8 will automatically hold. Given the parameter choices stated above in this section, we find by simulation that $N(0)_{\tau}>0$ for $\tau \in\left(0, \tau_{1}\right)$ (see Figure 4). For any particular $\tau \in\left(0, \tau_{1}\right)$, we can therefore define $N_{1}$ to be any positive constant such that $N_{1}<N(0)_{\tau}$. Condition (B1) of Theorem 8 will now hold. We need not specify $N_{1}$ in more precise terms to perform our simulations.

For $\tau \in\left(0, \tau_{1}\right)$, we define $h(\tau)$ as in (39) and we recall (second paragraph, section 9) that the sign of $h(\tau)$ determines the long-term influence of the culling regime of Theorem 8, with the mean pest population increased if $h(\tau)>0$ and decreased if $h(\tau)<0$. A plot of $h(\tau)$ against $\tau$, for $\tau \in\left(0, \tau_{1}\right)$, is given in figure 4 where we have used the parameter choices stated earlier in this section in order to create the plot. Notice that this plot confirms the observations of Section 9 - there exists $\tau^{*}$ such that $0<\tau^{*}<\tau_{1}$ where $h(\tau)<0$ and $N(0)_{\tau}>0$ for $\tau \in\left(0, \tau^{*}\right)$ (see (43)), and there exists $\tau^{* *}$ such that $\tau^{*}<\tau^{* *}<\tau_{1}$ where $h(\tau)>0$ and $N(0)_{\tau}>0$ for $\tau \in\left(\tau^{* *}, \tau_{1}\right)$ (see $(44)$ ). For the particular example shown in figure 4 we can choose $\tau^{*}$ and $\tau^{* *}$ to equal the single root $\tau_{r}$ of $h(\tau)=0$, namely $\tau_{r}=0.2560$.

If we make the same parameter choices that are made in Figure 4 and that are also stated earlier in this section, then we see that the culling regime of Theorem 8 will decrease the mean population if $\tau \in(0,0.2560)$ and will increase it if $\tau \in$ $(0.2560,2.8768)$. It is obviously worth demonstrating explicitly how the population is influenced by the culling regime, so we do this for two values of $\tau$ in Figure 5 . In the left plot of Figure 5 we have $\tau=0.06$, so we know that the culling regime will decrease the mean population, and indeed the plot clearly confirms this. In the right plot of Figure 5 we have $\tau=2$, so we know that the regime will increase the mean population, and this is confirmed by the plot. The two plots further corroborate Theorem 8 in that they show that the culling regime forces the population $N(t)$ to be periodic with period $2 \tau$.

The right plot of Figure 5 bears some resemblance to the plot involving a Nicholson birth function in Figure 1, although there are differences too - the shapes of the peaks in the right plot of Figure 5 are almost mirror images of the peaks in Figure 1.

In spite of Figure 4, it should not be assumed that $N_{0}(\tau)$ is always positive when 


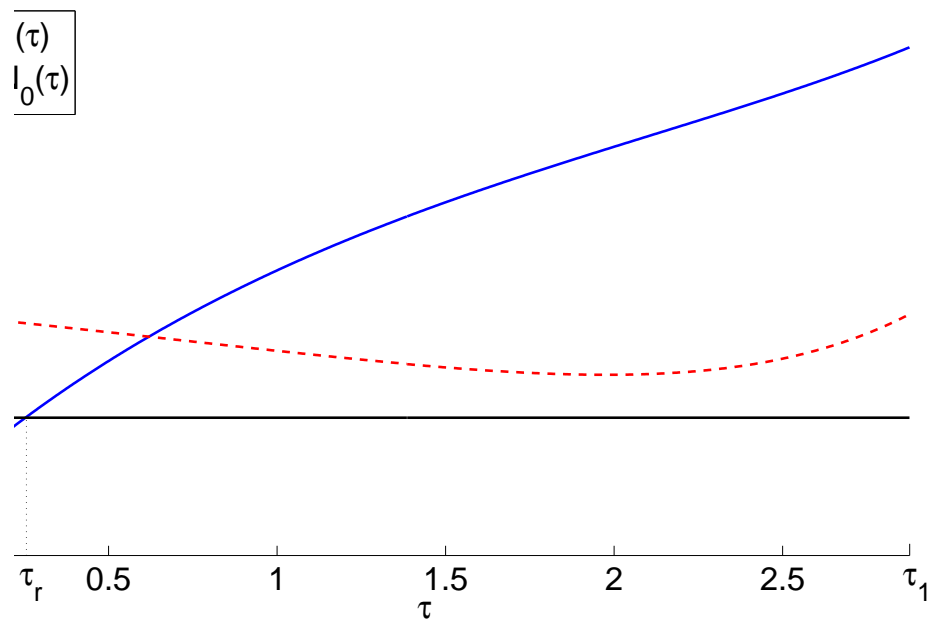

Figure 4: A plot of $h(\tau)=e^{\mu_{j} \tau} N^{*}(\tau)-\frac{M}{\mu}$ (see equation $(39)$ ) and $N_{0}(\tau)$ (see equation (36)). As explained in the third and fourth paragraphs of section 10, the conditions of theorem 8 hold when $N(0)_{\tau}>0$, and the culling regime of theorem 8 increases the mean pest population when $\tau$ is such that $h(\tau)>0$ and decreases it when $\tau$ is such that $h(\tau)<0$. Parameter choice here: $N_{2}=1.5, M=2, W=10$, $\mu=1$, and $\mu_{j}=0.1$. In the plot, $\tau_{1}=2.8768, \tau_{r}=0.2560$, and $h\left(\tau_{r}\right)=0$.

$\tau \in\left(0, \tau_{1}\right)$ or that $h(\tau)$ is always monotonic increasing for $\tau \in\left(0, \tau_{1}\right)$. Indeed, if we retain the parameter choices of Figure 4 except for our choice of $N_{2}$, which we reduce from 1.5 to 1 , then $N_{0}(\tau)$ becomes negative on the interval $0<\tau<\tau_{1}$ and $h(\tau)$ becomes decidedly non-monotonic, as we see in Figure 6 . When we bear in mind that Theorem 8 does not hold unless $N_{0}(\tau)>0$, Figure 6 shows that the theorem is valid for $\tau \in(0,0.7774)$ and for $\tau \in(6.9216,6.9315)$. Bearing in mind that the culling regime of Theorem 8 decreases the mean population when $h(\tau)<0$ and increases it when $h(\tau)>0$, Figure 6 therefore also shows that the regime decreases the mean population for $\tau \in(0,0.6027)$, increases it for $\tau \in(0.6027,0.7774)$, and increases it for $\tau \in(6.9216,6.9315)$. Thus, the precise relationship between the influence of culling on the mean population and the size of the maturation age $\tau$ is not, in general, trivially determined. We will explore this matter further in future research.

\section{Discussion}

We have examined a model for a creature with two life stages, namely juvenile and adult, where the adult stage is subject to culling. The length of the juvenile stage was assumed to be a constant $\tau$, which we called the maturation age. Motivated by simulations which suggest that infrequent but regular impulsive culling can, perversely, increase the average adult population, we sought an understanding of when such a result can hold analytically. Having established conditions such that periodic (but not necessarily impulsive) culling will reduce the mean population, we considered why culling may sometimes benefit a pest in the context of the shape of the birth function. This led us to construct a "step" birth function, which was simple but which retained the features that we felt would permit certain culling regimes to benefit a pest. 

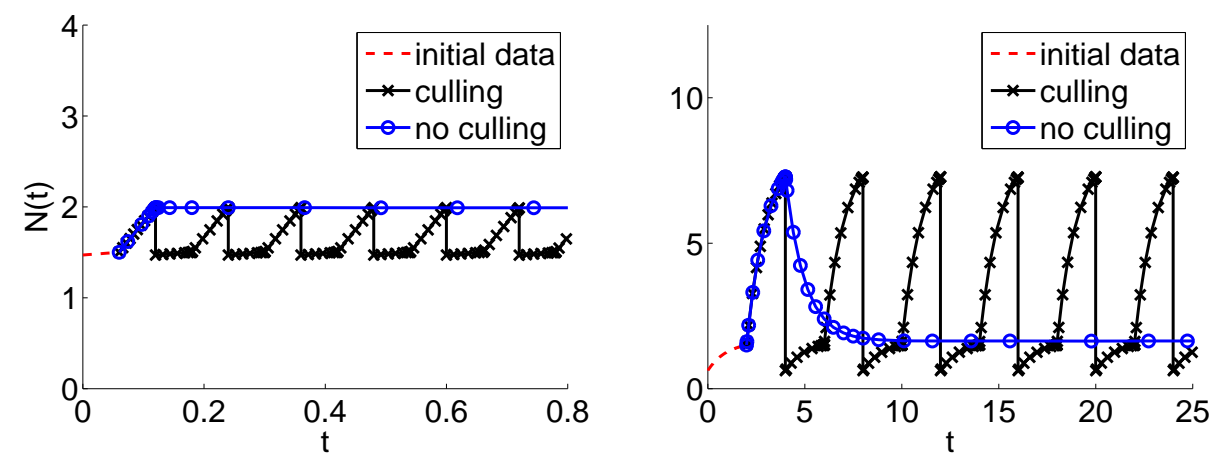

Figure 5: Numerical integration of the model for the adult population in the presence of culling (see assumption (A3) at the start of section 8) and in the absence of culling (see assumption (A2) at the start of section 8). The birth function is a step birth function, defined as in assumption (A1) at the start of section 8, and the initial data is defined as in (24). Left: culling decreases the mean. Model parameters: $\mu_{j}=0.1, \mu=1, N_{2}=1.5, M=2, W=10, N_{3}$ can be anything bigger than $\frac{W}{\mu}=10, \tau=0.06, N(0)=e^{-\mu_{j} \tau} \frac{M}{\mu}+e^{\mu \tau}\left(N_{2}-e^{-\mu_{j} \tau} \frac{M}{\mu}\right)=1.4698$, and $N_{1}$ can be anything less than or equal to $N(0)$. Culling regime is as defined in theorem 8: the first cull occurs at time $t_{1}=2 \tau=0.12$, culls occur thereafter every $T=2 \tau=0.12$ time units, and all culls have strength $\delta=0.2620$, which is found from equation (26). Right: culling increases the mean. Model parameters: Same as left picture except for $\tau=2$ and $N(0)=e^{-\mu_{j} \tau} \frac{M}{\mu}+e^{\mu \tau}\left(N_{2}-e^{-\mu_{j} \tau} \frac{M}{\mu}\right)=0.6218$. Culling regime is as defined in theorem 8: the first cull occurs at time $t_{1}=2 \tau=4$, culls occur thereafter every $T=2 \tau=4$ time units, and all culls have strength $\delta=0.9146$, which is found from equation (26). 


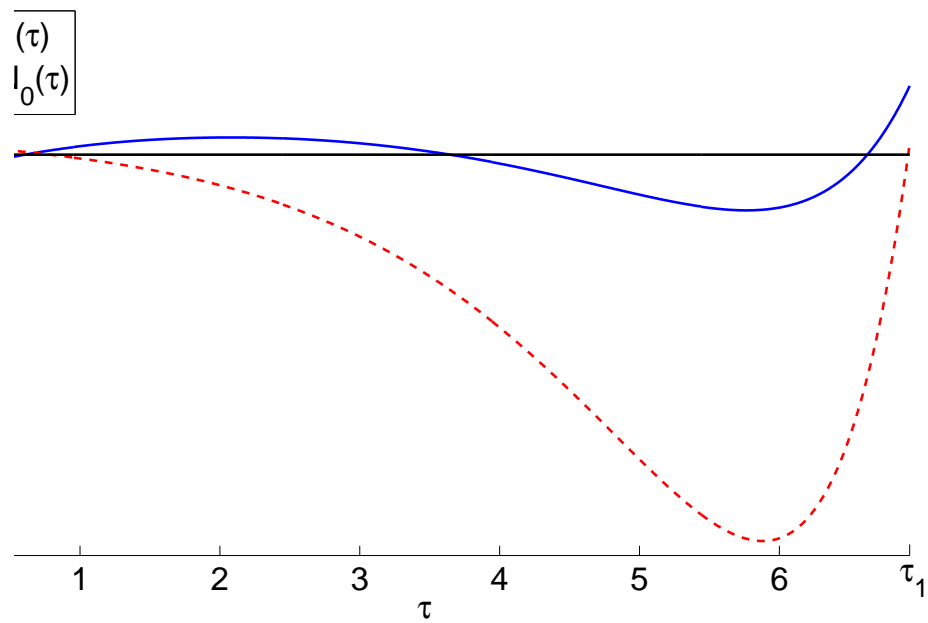

Figure 6: A plot of $h(\tau)=e^{\mu_{j} \tau} N^{*}(\tau)-\frac{M}{\mu}$ (see equation $(39)$ ) and $N_{0}(\tau)$ (see equation (36)). As explained in the third and fourth paragraphs of section 10, the conditions of theorem 8 hold when $N(0)_{\tau}>0$, and the culling regime of theorem 8 increases the mean pest population when $\tau$ is such that $h(\tau)>0$ and decreases it when $\tau$ is such that $h(\tau)<0$. Parameter choice here: $N_{2}=1, M=2, W=10$, $\mu=1$, and $\mu_{j}=0.1$. In the plot, $\tau_{1}=6.9315 ; h(\tau)=0$ where $\tau=0.6027,3.6373$, and 6.6294; and $N(0)_{\tau}=0$ where $\tau=0.7774$ and 6.9216 .

We recognised that there are circumstances under which a step birth function can be biologically realistic and the behaviour of a model with such a birth function was analysed in the absence of culling. We also constructed a specific culling regime and used it to demonstrate analytically that, for a certain positive finite range of values for the maturation age $\tau$, the regime increases the long-term mean pest population. We proved additionally that the same regime decreases the mean population if the maturation age $\tau$ is sufficiently small. Simulations corroborated our theoretical results.

The models in this paper may be appropriate to an insect crop pest subject to adult-stage culling by a pesticide. The drawbacks associated with large-scale applications of pesticides are numerous and include pesticide resistance, secondary pest outbreaks, and environmental degradation [13]. Yet results in this paper suggest that applying pesticides relatively infrequently can benefit a pest. We are in a position to conclude that both heavy use and light use of pesticides can ultimately work against us. But this is not to say that pesticides will always work against us. After all, we have shown that if the maturation age is suitably small then infrequent culling can be detrimental to the pest. In deciding whether or not to implement a pesticide program, we would recommend that simulations be performed to check that the regime will not increase the population as well as recommend that the environmental impact of the program be taken into account.

The models in this paper may also be appropriate to a large mammal subject to adult culling (by shooting) with a view to maintaining balances within its ecosystem. The culling of such an animal is likely to be infrequent because the intention would not be to eradicate it. However, since we have shown that infrequent impulsive culling can, in certain circumstances, increase the average population, we would recommend that, before implementing a culling regime for a large mammal, simulations be performed to check that the regime will not increase the population. 
Pulse vaccination strategies are routinely applied in many countries to control the spread of infectious diseases $[29,30]$. Theoretically analogous to the idea of impulsive culling benefiting a pest is the idea that pulses in a pulse vaccination strategy can sustain disease levels above their natural levels, a phenomenon encountered by Choisy et al [31]. Choisy et al demonstrate this phenomenon by simulation. We are not aware of any analytical demonstration in the literature. There would certainly be value in constructing a rigorous demonstration.

We have restricted our attention to periodic culling regimes but real-world control programs need not be periodic. For example, a farmer may choose to apply a pesticide only at those particular times when a pest appears to be especially abundant. Thus, as future research, we may investigate non-periodic culling regimes. Our analytical demonstration that infrequent culling can increase the average population of a pest was limited to a model with a step birth function and adult impulsive culling. There would be value in seeking a more general set of conditions under which adult culling benefits a pest. Finally, all of our regimes have involved culling only the adults. But juveniles can be culled as well - in the case of insects, larvicides are commonly applied. As future work, then, we may study juvenile-stage culling with a view to proving that it too can benefit a pest.

\section{References}

[1] C. J. Bolter, M. Dicke1, J. J. A. van Loon, J. H. Visser, and M. A. Posthumus, Attraction of Colorado Potato Beetle to herbivore-damaged plants during herbivory and after its termination, Journal of Chemical Ecology, 23:4 (1997) 1003-1023.

[2] K. Seok Kim and T. W. Sappington, Boll Weevil (Anthonomus grandis Boheman) (Coleoptera: Curculionidae) dispersal in the Southern United States: evidence from mitochondrial DNA variation, Environmental Entomology 33:2 (2004) 457-470.

[3] P. Witzgall, L. Stelinski, L. Gut, and D. Thomson, Codling moth management and chemical ecology, Annual Review of Entomology, 53 (2008) 503-522.

[4] D. J. Gubler, Resurgent vector-borne diseases as a global health problem. Emerging Infectious Diseases, 4:3 (1998) 442-450.

[5] P. R. Epstein, Is Global Warming Harmful to Health? Scientific American, 283 (2000) $50-57$.

[6] C. A. Donnelly, R. Woodroffe, D. R. Cox, F. J. Bourne, C. L. Cheeseman, R. S. Clifton-Hadley, G. Wei1, G. Gettinby, P. Gilks, H. Jenkins, W. T. Johnston, A. M. Le Fevre1, J. P. McInerney, and W. I. Morrison, Positive and negative effects of widespread badger culling on tuberculosis in cattle, Nature, 439 (2006) 843-846.

[7] V. M. Trenkel, Exploring red deer culling strategies using a population-specific calibrated management model, Journal of Environmental Management, 62:1 (2001) 37-53.

[8] R. van Aarde, I. Whyte, and S. Pimm, Culling and the dynamics of the Kruger National Park African elephant population, Animal Conservation, 2 (1999) 287-294.

[9] I. J. East and C. H. Eisemann, Vaccination against Lucilia cuprina: The causative agent of sheep blowfly strike, Immunology and Cell Biology, 71 (1993) 453-462.

[10] B. L. Phillips, G. P. Brown, and R. Shine, Assessing the potential impact of cane toads on Australian snakes, Conservation Biology, 17:6 (2003) 1738-1747.

[11] B. L. Phillips, G. P. Brown, J. K. Webb, and R. Shine, Invasion and the evolution of speed in toads, Nature, 439 (2006) 803.

[12] R. R. L. Simons and S. A. Gourley, Extinction criteria in stage-structured population models with impulsive culling, SIAM Journal of Applied Mathematics, 66:6 (2006) 1853-1870.

[13] A. J. Terry, Impulsive adult culling of a tropical pest with a stage-structured life cycle, in press in Nonlinear Analysis: Real World Applications, 2009. DOI: 10.1016/j.nonrwa. 2009.01.005. 
[14] G. T. Miller, Sustaining the Earth, Sixth edition, pp. 211-216, Chapter 9, Thompson Learning, Inc. Pacific Grove, California, 2004.

[15] L. A. Lacey, R. Frutos, H. K. Kaya, and P. Vail, Insect pathogens as biological control agents: do they have a future?, Biological Control, 21 (2001) 230-248.

[16] A. F. G. Dixon, Aphid Ecology: An Optimization Approach, Chapman and Hall, 1998.

[17] K. Cooke, P. van den Driessche \& X. Zou, Interaction of maturation delay and nonlinear birth in population and epidemic models. J. Math. Biol. 39 (1999), 332-352.

[18] A. J. Nicholson, An outline of the dynamics of animal populations, Australian Journal of Zoology, 2 (1954) 9-65.

[19] A. J. Nicholson, The self-adjustment of populations to change, Cold Spring Harbour Symposium on Quantitative Biology, 22 (1957) 153-173.

[20] W. Gurney, S. Blythe, and R. Nisbet, Nicholson's blowflies revisited, Nature, 287 (1980) $17-21$.

[21] W. E. Ricker, Stock and recruitment, J. Fisheries Res. Board Canada, 11 (1954) 559-623.

[22] W. E. Ricker, Handbook of Computations for Biological Statistics of Fish Populations, Bull. Fisheries Res. Board Canada, 119, 1958.

[23] H. L. Smith, Monotone Dynamical Systems: An Introduction to the Theory of Competitive and Cooperative Systems, American Mathematical Society, Providence, Rhode Island, 1995.

[24] S. A. Gourley, R. Liu, and J. Wu, Eradicating vector-born diseases via age-structured culling, Journal of Mathematical Biology, 54:3 (2007) 309-335.

[25] H. R. Thieme, Mathematics in population biology. Princeton Series in Theoretical and Computational Biology. Princeton University Press, Princeton, NJ, 2003.

[26] Deimling, K. Nonlinear functional analysis. Springer-Verlag, Berlin, 1985.

[27] Zhao, X.-Q. Dynamical systems in population biology. CMS Books in Mathematics, Springer-Verlag, New York, 2003.

[28] Y. Kuang, Delay Differential Equations with Applications in Population Dynamics, Academic Press, Inc., 1993.

[29] World Health Organization Press Release $(2 / 11 / 2000)$, Pulse Polio Campaign Targets over 107 Million Children in 11 States, http://www . searo. who.int/EN/Section316/Section503/Section2373_12973.htm

[30] B. Shulgin, L. Stone, and Z. Agur, Pulse vaccination stategy in the SIR epidemic model, Bulletin of Mathematical Biology, 60 (1998) 1123-1148.

[31] M. Choisy, J-F. Guegán, and P. Rohani, Dynamics of infectious diseases and pulse vaccination: Teasing apart the embedded resonance effects, Physica D, 223 (2006) 26-35. 